Pacific

Journal of

Mathematics

\title{
BLOWUPS AND GAUGE FIELDS
}

Nicholas P. Buchdahl 


\title{
BLOWUPS AND GAUGE FIELDS
}

\author{
NiChOLAS P. BuChDAHL
}

The relationship between stable holomorphic vector bundles on a compact complex surface and the same such objects on a blowup of the surface is investigated, where "stability" is with respect to a Gauduchon metric on the surface and naturally derived such metrics on the blowup.

The main results are: descriptions of holomorphic vector bundles on a blowup; conditions relating (semi)-stability of these to that of their direct images on the surface; sheaftheoretic constructions for "stabilizing" unstable bundles and desingularising moduli of stable bundles; an analysis of the behavior of Hermitian-Einstein connections on bundles over blowups as the underlying Gauduchon metric degenerates; the definition of a topology on equivalence classes of stable bundles on blowups over a surface and a proof that this topology is compact in many cases.

\section{Introduction.}

The purpose of this paper is to investigate the relationship between between stable holomorphic vector bundles on a compact complex surface and the same such objects on a modification (blowup) of the surface. In large part, the paper is a continuation of the work in [B3] where it was shown that a holomorphic bundle on a compact complex surface admits an irreducible Hermitian-Einstein connection if and only if the bundle is stable, the notions of stability and Hermitian-Einstein both being with respect the same $\bar{\partial} \partial-$ closed positive (1,1)-form: This is a generalization of Donaldson's result [D2] where it is assumed that the form is $d$-closed and defines an integral cohomology class (i.e., the algebraic case).

Much of the underlying motivation for this work comes from its potential applications to topology (though no such applications are considered here). Donaldson has proved fundamental results on the topology of smooth 4manifolds by defining topological invariants of moduli spaces of solutions of the anti-self-dual Yang-Mills equations on a Riemannian 4-manifold, and showing that these are differential invariants of the manifold itself. In the case of an algebraic surface with Hodge metric, the result of [D2] mentioned above identifies the Yang-Mills moduli spaces with moduli spaces of stable 
vector bundles, and these spaces and/or invariants can be computed using techniques standard in complex analysis. In this way Donaldson has been able to prove some of his most remarkable results [D4], [D5], and others have built upon his work ([FM1], [FM2], $[\mathbf{K 1}],[\mathbf{O V}]$, to cite just a few. See also [FM3] for a comprehensive account of developments, and [FS] for a calculation of the Donaldson invariants of a blown-up 4-manifold in terms of those of the manifold). This interaction between real analysis, complex analysis and topology provides a rich area for investigation, and parts of this paper are directly concerned particularly with the interplay between the real and the complex analysis.

The proof of the main result of [B3] is a modification of that given by Donaldson [D1] to prove the same theorem in the case of Riemann surfaces. The differences in the proofs arise from the appearance of certain singularities in the two-dimensional case, and a successful way around these singularities is to blow up the surface and pull back. In so doing, various relationships between bundles and sheaves on the surface and its blowups are uncovered, and these relationships turn out to be directly related to other aspects of gauge theory and/or complex analysis which are themselves of independent interest.

A study of degenerating sequences of stable bundles on the projective plane leads naturally to conjecture whether blowups can be used to compactify moduli spaces of stable bundles in general. In [B5], it is shown that sequences of stable bundles, identified with sequences of Hermitian-Einstein connections have convergent subsequences after pulling back to blowups, at least when weak limits are stable. This leads to the definition of a natural topology on moduli spaces of stable bundles over a surface and its blowups, and the proof of the compactness of the generic such space is presented here.

The paper is organized as follows: $\S 1$ introduces notation, definitions and central background material, and gives some useful lemmas concerning "invariants" of stable holomorphic bundles. In $\S 2$ a local description and characterization of bundles on the blowup of the ball in $\mathbb{C}^{2}$ at the origin is given. A holomorphic version of Taubes' "cut-and-paste" construction for gauge fields $[\mathbf{T}]$ is given, enabling a global description of bundles on the blowup of an arbitrary complex surface in terms of bundles on the original surface. Also included in this section is a short discussion of the relationship to - and between - associated constructions of Serre and Schwarzenberger.

Questions of stability are considered in the third section from a purely complex-analytic viewpoint, and a detailed description of the conditions required for bundles on a blowup $\widetilde{X} \stackrel{\pi}{\rightarrow} X$ to be stable is given. Of course, the metric on $\widetilde{X}$ with respect to which stability is considered must be specified, and the fore-mentioned conditions are as much on the metric as on the bundles themselves. From a real analytical view-point, it turns out the correct 
metrics are those formed from the original metric on $X$ together with the Fubini-Study metric on $\overline{\mathbb{P}}_{2}$ combined so as to "stretch-out" the neck of the connected sum $\widetilde{X} \simeq_{\text {diffeo }} X \# \overline{\mathbb{P}}_{2}$, and the main result of this section shows that once the neck is sufficiently long, the moduli spaces effectively become independent of the metric.

The analysis in the third section encounters pathological sheaves which are semi-stable but not stable. Using the cut-and-paste method, a mechanism for "stabilizing" such sheaves is given in $\S 4$. A similar method also provides a simple way to desingularise singular points in moduli spaces.

For a bundle in one of the "stable" moduli spaces of $\S 3$, the behavior of the corresponding sequence of Hermitian-Einstein connections as the metrics degenerate is investigated in $\S 5$.

In the last section, the issue of compactness for moduli spaces of stable bundles is considered. In [B5] it is shown that after sufficiently many blowups and pull-backs, sequences of stable bundles of bounded topology and degree have strongly convergent subsequences, where stable bundles are identified with irreducible Hermitian-Einstein connections. A natural candidate for a compactification of a moduli space of stable bundles as presented in $[\mathbf{B 5}]$ is shown, under generic conditions in the arbitrary rank case, and in general for the rank 2 case, to be a compact space; some other simple properties of this space are also considered.

Acknowledgments. Parts of this paper were written in 1988 when I was a visiting member of the Institute for Advanced Study, Princeton, N. J. supported by the National Science Foundation. Other parts were written while a member of the Department of Mathematics at Tulane University, New Orleans with the support of NSF Grant \#DMS 8900878. The work was completed while visiting the Department of Mathematics at the University of Nantes, to which institution I am very grateful for its hospitality. I am also grateful to the École Polytechnique, Paris for its hospitality during the same period, where further additions to the paper were made.

\section{Preliminaries.}

The purpose of this section is to re-cap on, and to expand upon the basic notation, definitions and results of [B3]. Further details can be found in that reference.

Let $X$ be a compact complex surface and let $\omega$ be a $\bar{\partial} \partial$-closed positive $(1,1)$-form on $X$ : It is a theorem of Gauduchon [Gau] that every positive $(1,1)$-form has a conformal rescaling such the rescaled form is $\bar{\partial} \partial$-closed. The conformal factor is unique up to positive constants and is completely determined by requiring that the new form give the same volume $V:=$ $\operatorname{Vol}(X, \omega):=\frac{1}{2} \int_{X} \omega^{2}$. With such a form $\omega$, the degree $\operatorname{deg}(L)=\operatorname{deg}(L, \omega)$ of 
a holomorphic line bundle $L$ on $X$ is unambiguously defined by the formula

$$
\operatorname{deg}(L):=\frac{i}{2 \pi} \int_{X} f_{L} \wedge \omega
$$

where $f_{L}$ is the curvature of any hermitian connection on $L$. The degree depends only on $c_{1}(L)$ if and only if $b_{1}(X)$ is even, and when this is the case $\omega$ is cohomologous modulo the image of $\partial+\bar{\partial}$ to a closed form which itself is unique up to the image of $\bar{\partial} \partial$; ([B3, Proposition 2]).

If $E$ is a holomorphic $r$-bundle on $X$, set $\operatorname{deg}(E):=\operatorname{deg}(\operatorname{det} E)$ and $\mu(E):=\operatorname{deg}(E) / r$; the latter is called the normalized degree or slope of $E$. A hermitian connection on $E$ is Hermitian-Einstein if the curvature $F$ satisfies $\widehat{F}=i \lambda \mathbf{1}$ where $\widehat{F}:=*(\omega \wedge F)=: \Lambda F, \lambda=(-2 \pi / V) \cdot \mu(E)$ and 1 is the identity endomorphism of $E$. The bundle $E$ is (semi-) stable if $\mu(\mathcal{S})<(\leq) \mu(E)$ for every coherent subsheaf $\mathcal{S} \subset E$ with $0<\operatorname{rank}(\mathcal{S})<$ $r$. As mentioned in the introduction, the main result of [B3] is that a bundle admits an irreducible Hermitian-Einstein connection if and only if it is stable, this generalizing the same result proved by Donaldson [D2] in the case that $(X, \omega)$ is algebraic. A bundle admitting a Hermitian-Einstein connection is a direct sum of stable bundles all of the same normalized degree; i.e., is quasi-stable.

If $E$ has a Hermitian-Einstein connection with curvature $F$, the equation $\omega \wedge\left(F-\frac{1}{r} \operatorname{tr} F \mathbf{1}\right)=0$ and the skew-Hermitian property of $F$ give $\operatorname{tr}(F-$ $\left.\frac{1}{r} \operatorname{tr} F \mathbf{1}\right)^{2}=\left|F-\frac{1}{r} \operatorname{tr} F \mathbf{1}\right|^{2} d V$. Since the former 4 -form is a representative for the characteristic class $8 \pi^{2}\left(c_{2}-\frac{r-1}{2 r} c_{1}^{2}\right)(E)$, this motivates defining the charge of $E, C(E)$, for an arbitrary $r$-bundle $E$ by the formula

$$
C(E):=\left(c_{2}-\frac{r-1}{2 r} c_{1}^{2}\right)(E)=\frac{1}{8 \pi^{2}} \int_{X} \operatorname{tr}\left(F-\frac{1}{r} \operatorname{tr} F \mathbf{1}\right)^{2} .
$$

This number is non-negative for any bundle admitting a Hermitian-Einstein connection, and when this is the case, is identically zero only if the induced Hermitian-Einstein connection on the adjoint bundle is flat; (cf. $[\mathbf{L}])$. Note that the charge is invariant under tensoring by line bundles: $C(E \otimes L)=$ $C(E)$ for any such $L$. In general, $C(E \otimes A)=a C(E)+r C(A)$, where $a, r$ are the ranks of $A, E$ respectively.

Recall that a coherent analytic sheaf $\mathcal{S}$ is torsion-free if and only if the canonical morphism $\mathcal{S} \rightarrow \mathcal{S}^{* *}$ is injective, and $\mathcal{S}$ is by definition reflexive if this map is an isomorphism; recall also that the singularity sets of such sheaves are of codimension at least 2 and 3 respectively; ([OSS, II.1.1]). For exact sequences $0 \rightarrow \mathcal{A} \rightarrow \mathcal{B} \rightarrow \mathcal{C} \rightarrow 0$ of locally free sheaves on $X$ it is easy to check that the charges are related by

$$
C(\mathcal{B})=C(\mathcal{A})+C(\mathcal{C})-\frac{b}{2 a c}\left[\frac{a}{b} c_{1}(\mathcal{B})-c_{1}(\mathcal{A})\right]^{2},
$$


where $a, b$ and $c$ are the ranks of $\mathcal{A}, \mathcal{B}$ and $\mathcal{C}$ respectively. The definition of charge extends to torsion-free sheaves $\mathcal{S}$ of rank $r$ by means of the formula

$$
C(\mathcal{S}):=C\left(\mathcal{S}^{* *}\right)+h^{0}\left(\mathcal{S}^{* *} / \mathcal{S}\right)
$$

which is consistent with a definition of $c_{2}(\mathcal{S})$ extending that of the Chern character on bundles in such a way that the Hirzebruch-Riemann-Roch formula

$h^{0}(\mathcal{S})-h^{1}(\mathcal{S})+h^{2}(\mathcal{S})=\chi(\mathcal{S})=-C(\mathcal{S})+\frac{1}{2 r} c_{1}^{2}(\mathcal{S})+\frac{1}{2} c_{1}(\mathcal{S}) \cdot c_{1}(X)+r \chi\left(\mathcal{O}_{X}\right)$

remains valid. If $\mathcal{C}$ is only torsion-free, it follows from this definition that (1.2) remains valid and this in turn implies that the formula (1.2) holds for arbitrary torsion-free sheaves $\mathcal{A}, \mathcal{B}$ and $\mathcal{C}$. Note that a torsion-free sheaf is (semi-)stable iff its double-dual is.

If $b_{1}(X)$ is odd, the intersection form on $H^{2}(X, \mathbb{R})$ restricted to $H^{1,1}(X)$ is negative definite ([BPV, Theorem IV.2.13]) and the last term on the right in (1.2) therefore contributes positively to the sum. If $b_{1}(X)$ is even, the intersection form restricted to $H^{1,1}(X)$ has one positive eigenvalue and the rest are all negative. In either case, $\omega$ defines a positive definite hermitian form on $H^{1,1}(X)$ by setting $\|f\|^{2}:=V^{-1}|(f, \omega)|^{2}-(f, f)$, where $(f, g):=$ $\int_{X} \bar{f} \wedge g$; (recall $V=(\omega, \omega) / 2$ throughout). Equation (1.2) can therefore be written

$$
C(\mathcal{B})=C(\mathcal{A})+C(\mathcal{C})+\frac{b}{2 a c}\left\|\frac{a}{b} c_{1}(\mathcal{B})-c_{1}(\mathcal{A})\right\|^{2}-\frac{b}{2 a c} \frac{\nu_{\mathcal{B}}(\mathcal{A})^{2}}{V},
$$

where $\nu_{\mathcal{B}}(\mathcal{A}):=a[\mu(\mathcal{B})-\mu(\mathcal{A})]$. By induction on rank, it follows the charge is non-negative for any torsion-free semi-stable sheaf. Note that if $b_{1}(X)$ is odd it follows by induction from (1.2) (and the existence of HermitianEinstein connections on stable bundles) that the charge is non-negative for any torsion-free coherent analytic sheaf, semi-stable or otherwise.

The function $\nu_{\bullet}(*)$ plays an important role in the proof of the main result of [B3]. It has a number of simple but useful properties, three of which are summarised for convenience in the following lemma.

\section{Lemma 1.5.}

(a) If

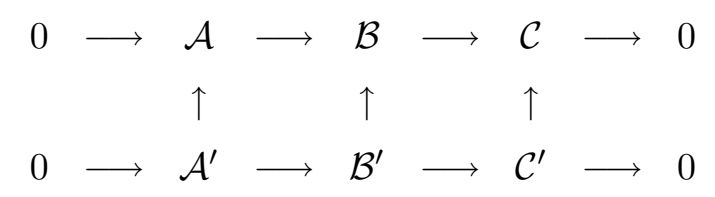


is a commutative diagram with exact rows such that the vertical arrows are inclusions, then

$$
\nu_{\mathcal{B}}\left(\mathcal{B}^{\prime}\right)=\nu_{\mathcal{A}}\left(\mathcal{A}^{\prime}\right)+\nu_{\mathcal{C}}\left(\mathcal{C}^{\prime}\right)+\left(\frac{a^{\prime}}{a}-\frac{c^{\prime}}{c}\right) \nu_{\mathcal{B}}(\mathcal{A})
$$

(b) If $\mathcal{A}$ and $\mathcal{B}$ are locally free and if $\mathcal{B}$ is stable and $\mathcal{A} \subset \mathcal{B}$ minimizes $\nu_{\mathcal{B}}$ over all non-zero subsheaves of $\mathcal{B}$ of strictly smaller rank then $\mathcal{A}$ is stable and in addition, the quotient $\mathcal{C}=\mathcal{B} / \mathcal{A}$ is both torsion-free and stable;

(c) If $E$ is a holomorphic bundle equipped with a Hermitian metric and $A \subset E$ has torsion-free quotient $C$ then off the singular set of $C$ the second fundamental form $\beta \in \Lambda^{0,1} \otimes \operatorname{Hom}(C, A)$ of the induced Hermitian connection lies in $L^{2 p}(X)$ for any $p<2$, and

$$
\nu_{E}(A)=-\frac{1}{2 \pi} \int_{X} \operatorname{tr}_{A}\left(i F(E)+\lambda_{E} \mathbf{1} \omega\right) \wedge \omega+\|\beta\|_{L^{2}(\omega)}^{2} .
$$

The proof of (c) is given in [B3] (Remark (f) p. 634). Part (b) is the same as Lemma 2 of the same reference; the proof follows immediately from (1.6) which itself is a straight-forward calculation. The existence of such $\mathcal{A} \subset \mathcal{B}$ minimizing $\nu_{\mathcal{B}}$ when the latter is stable is proved in Lemma 4 of [B3], which provides one of the key steps in the proof of the main result there by enabling the argument to proceed by induction on rank; it is also proved in that lemma that there always exists $\mathcal{A} \subset \mathcal{B}$ maximizing $\mu$ over admissible subsheaves regardless of the stability or otherwise of $\mathcal{B}$, admissible meaning that it is coherent and has positive rank strictly less than that of $\mathcal{B}$. For a stable bundle $E$ it follows that $\nu_{E}(*)$ is positive and bounded away from 0 on the set of admissible subsheaves of $E$, from which it follows immediately that stability is an open condition on the metric.

When $b_{1}(X)$ is even, this bound on the slopes of subsheaves can be made uniform in $E$ as the next result shows:

Lemma 1.8. Suppose that $b_{1}(X)$ is even. For any $r_{0}, C_{0}>0$ there exists $\delta_{0}=\delta_{0}\left(r_{0}, C_{0}\right)>0$ with the following property: If $E$ is a semi-stable torsionfree sheaf of rank $r \leq r_{0}$ and charge $C(E) \leq C_{0}$ admitting a subsheaf $A \subset E$ with $\nu_{E}(A)<\delta_{0}$, then $\nu_{E}(A)=0$.

Proof. Since $C\left(E^{* *}\right) \leq C(E)$ and $\nu_{E^{* *}}\left(A^{* *}\right)=\nu_{E}(A)$ it suffices to prove the result with "torsion-free" in the hypotheses replaced by "locally free". For subsheaves $A \subset E$ of the same rank as $E$ the result follows from Corollary 2 of [B3], so it can also be supposed that all such subsheaves have rank strictly less than that of the ambient bundle.

If there is no stable bundle of rank $\leq r_{0}$ and charge $\leq C_{0}$ which admits a proper non-zero reflexive subsheaf, then $\nu_{E}$ is identically 0 for all such bundles. Otherwise, there is a sequence $\left\{E_{i}\right\}$ of bundles admitting such subsheaves $A_{i} \subset E_{i}$ with $\left\{\nu_{E_{i}}\left(A_{i}\right)\right\}$ strictly decreasing. By Lemma 4 of 
[B3], it can be assumed that each $A_{i}$ minimizes $\nu_{E_{i}}$ over the proper nonzero subsheaves of $E_{i}$, and by Lemma 1.5(b), both $A_{i}$ and the quotient $C_{i}:=E_{i} / A_{i}$ are torsion-free and stable. Since $C\left(A_{i}\right)$ and $C\left(C_{i}\right)$ are therefore non-negative and $\nu_{E_{i}}\left(A_{i}\right)=\left|\nu_{E_{i}}\left(A_{i}\right)\right|$ is decreasing, (1.4) and the bound on the rank and charge of $E_{i}$ give a uniform bound on $\left\|a_{i} c_{1}\left(E_{i}\right)-r_{i} c_{1}\left(A_{i}\right)\right\|$, so there is a subsequence with $r_{i}$ and $a_{i} c_{1}\left(E_{i}\right)-r_{i} c_{1}\left(A_{i}\right)$ constant. Since $b_{1}(X)$ is even, the degree is topological so $\nu_{E_{i}}\left(A_{i}\right)$ is constant on the subsequence, implying that the original sequence is finite. It follows that there exists $\delta>0$ such that $\nu_{E}(A) \geq \delta$ for any proper non-zero subsheaf $A$ of a stable torsion-free sheaf $E$ of rank $\leq r_{0}$ and charge $\leq C_{0}$. Set $\delta_{0}:=\delta$.

If now $E$ is semi-stable (of rank $\leq r_{0}$ and charge $\leq C_{0}$ ) and $D \subset E$ satisfies $\nu_{E}(D)<\delta_{0}$, then $E$ cannot be stable so there exists non-trivial $A \subset E$ with torsion-free quotient $C$ such that $\mu(A)=\mu(E)$. Since $E$ is semi-stable, so too are both $A$ and $C$; and, moreover, they also satisfy the hypotheses of the lemma by (1.4). If $C^{\prime}$ is the image of the composition $D \rightarrow E \rightarrow C$ and $A^{\prime}$ is the kernel, the result follows from (1.6) using induction on $\operatorname{rank}(E)$.

Remarks. The result is false if $b_{1}(X)$ is not even: Moduli spaces of stable 2 -bundles with trivial determinant on a Hopf surface are explicitly computed in $[\mathbf{B H}]$, and the description there shows that there are stable 2-bundles of charge 1 possessing subsheaves of degree arbitrarily close to 0 .

\section{Vector bundles on a blowup.}

In this section the nature of holomorphic vector bundles on a neighborhood of a blown-up point in a complex surface is investigated. Two approaches are taken: The first starts from the splitting type of such bundles on the exceptional divisor, whereas the second is more global in nature, identifying such bundles with a class of bundles on the complex projective plane.

Let $Y$ be a discrete set of points in a complex surface $X$ and let $\widetilde{X} \stackrel{\pi}{\rightarrow} X$ be the blowup of $X$ along $Y$. The exceptional divisor $\widetilde{Y}=\pi^{-1}(Y) \subset \widetilde{X}$ is defined by a section of a certain holomorphic line bundle, and since this line bundle restricts to $\mathcal{O}(-1)$ on each component of $\widetilde{Y}$ the notation $\mathcal{O}(-1)$ will be used to denote (the sheaf of sections of) this line bundle. If $\mathcal{I}_{Y} \subset \mathcal{O}_{X}$ denotes the ideal sheaf of $Y$ and $\mathcal{N}_{Y}=\left(\mathcal{I}_{Y} / \mathcal{I}_{Y}^{2}\right)^{*}$ is the normal bundle of $Y$ in $X$, then it is straightforward to show that the direct images of the sheaves 
$\mathcal{O}(n)$ under $\pi$ are canonically given by

$$
\begin{aligned}
& \pi_{*} \mathcal{O}_{\widetilde{X}}(n)= \begin{cases}\mathcal{I}_{Y}^{n} & \text { if } n \geq 0 \\
\mathcal{O} & \text { if } n \leq 0\end{cases} \\
& \pi_{*}^{1} \mathcal{O}_{\widetilde{X}}(n)= \begin{cases}0 & \text { if } n \geq-1 \\
\operatorname{det} \mathcal{N}_{Y} & \text { if } n=-2 \\
\mathcal{E} x t_{\mathcal{O}}^{1}\left(\mathcal{I}_{Y}^{-n-1}, \mathcal{O}\right) & \text { if } n \leq-2\end{cases}
\end{aligned}
$$

(where $\pi_{*}^{q}=R^{q} \pi_{*}$ denotes the $q$-th direct image under $\pi$ ) with all other direct images vanishing.

Let $U \subset X$ be a small ball in $X$ such that $U \cap Y$ is the singleton $\left\{x_{0}\right\}$, let $\widetilde{U} \stackrel{\pi}{\rightarrow} U$ be the blowup of $U$ at $x_{0}$, and let $L_{0}=\pi^{-1}\left(x_{0}\right)$ be the exceptional line. If $\widetilde{E}$ is a holomorphic $r$-bundle on $\widetilde{U}$, then the restriction of $\widetilde{E}$ to $L_{0}$ splits as a sum of line bundles, and the nature of this splitting determines much about the bundle itself, as will be demonstrated in the results which follow.

Lemma 2.2. Suppose $\left.\widetilde{E}\right|_{L_{0}}=\bigoplus_{i=1}^{r} \mathcal{O}\left(a_{i}\right)$.

(a) If $a_{i} \leq 0$ for all $i$ then $\pi_{*} \widetilde{E}$ is locally free;

(b) If $a_{i} \geq-1$ for all $i$ then $\pi_{*}^{1} \widetilde{E}=0$;

(c) If $a_{i}=0$ for all $i$ then $\widetilde{E}$ is trivial on $\widetilde{U}$ and $\widetilde{E}=\pi^{*} \pi_{*} \widetilde{E}$;

(d) If $\pi_{*} \underset{E}{\widetilde{E}}$ is locally free then $\sum_{i} a_{i} \leq 0$;

(e) If $\pi_{*}^{1} \widetilde{E}=0$ then $a_{i} \geq-1$ for all $i$;

(f) If $\pi_{*} \widetilde{E}$ is locally free and $\pi_{*}^{1} \widetilde{E}=0$ then $\widetilde{E}=\oplus_{i} \mathcal{O}(-1) \oplus \oplus_{j} \mathcal{O}(0)$.

Proof. The space $\widetilde{U}$ can be viewed as a closed subspace of $U \times \mathbb{P}_{1}$, defined by a section of the line bundle $\mathcal{O}(1)$. The bundle $\widetilde{E}$ on $\widetilde{U}$ can be extended (non-uniquely) to a bundle $\widetilde{E}^{\prime}$ on $U \times \mathbb{P}_{1}$ simply by extending a transition function on the intersection of a pair of Stein sets covering $\widetilde{U}$. Thus there is an exact sequence

$$
0 \rightarrow \widetilde{E}^{\prime}(-1) \rightarrow \widetilde{E}^{\prime} \rightarrow \widetilde{E} \rightarrow 0
$$

on $U \times \mathbb{P}_{1}$, (where the notation $\widetilde{E}(n)$ denotes $\widetilde{E} \otimes \mathcal{O}(n)$ throughout).

If $a_{i}<0$ for all $i$ then $H^{0}\left(\{x\} \times \mathbb{P}_{1}, \widetilde{E}^{\prime}\right)=0$ for $x=x_{0}$ and hence for all $x$ in a neighborhood of $x_{0}$ by semi-continuity of cohomology. From the basechange theorem $\left(\left[\mathbf{B S}\right.\right.$, Theorem 3.4]) it follows that $\pi_{*} \widetilde{E}^{\prime}=0=\pi_{*} \widetilde{E}^{\prime}(-1)$ and the sheaves $\pi_{*}^{1} \widetilde{E}^{\prime}$ and $\pi_{*}^{1} \widetilde{E}^{\prime}(-1)$ are locally free on $U$. From the direct image of $(2.3)$

$$
0 \rightarrow \pi_{*} \widetilde{E} \rightarrow \pi_{*}^{1} \widetilde{E}^{\prime}(-1) \rightarrow \pi_{*}^{1} \widetilde{E}^{\prime} \rightarrow \pi_{*}^{1} \widetilde{E} \rightarrow 0
$$


it therefore follows from Lemma II.1.1.10 of [OSS] that $\pi_{*} \widetilde{E}$ is reflexive, and hence locally free. Moreover, taking direct images of the exact sequence $\widetilde{E} \otimes\left(0 \rightarrow \mathcal{O}_{\widetilde{U}}(1) \rightarrow \mathcal{O}_{\widetilde{U}} \rightarrow \mathcal{O}_{L_{0}} \rightarrow 0\right)$ on $\widetilde{U}$ and using the fact that $\pi_{*}\left(\left.\widetilde{E}\right|_{L_{0}}\right)$ vanishes, it follows that $\pi_{*} \widetilde{E}(1)=\pi_{*} \widetilde{E}$ is also reflexive, which proves (a).

To prove (b), if $a_{i} \geq-1$ for all $i$ then $H^{1}\left(\{x\} \times \mathbb{P}_{1}, \widetilde{E}^{\prime}\right)$ vanishes at $x=x_{0}$, and therefore for all $x$ in a neighborhood of $x_{0}$, again by semi-continuity of cohomology. Taking direct images of (2.3) this time shows that $\pi_{*}^{1} \widetilde{E}$ vanishes near $x_{0}$.

To prove (c), if $\widetilde{E}$ is trivial on $L_{0}$, then $\pi_{*} \widetilde{E}$ is locally free by (a). The canonical sheaf homomorphism $\pi^{*} \pi_{*} \widetilde{E} \rightarrow \widetilde{E}$ is a bundle isomorphism off the exceptional divisor, but since the two bundles have the same determinant, it must be an isomorphism everywhere.

To prove (d), suppose $\operatorname{det} \widetilde{E}=\mathcal{O}(a)$ for some $a>0$. If $\pi_{*} \widetilde{E}$ is locally free, then by $(2.1)$ so too is $\pi_{*} \widetilde{E}^{\prime \prime}$ for $\widetilde{E}^{\prime \prime}:=\widetilde{E} \oplus \mathcal{O}(-a)$, and therefore $\pi^{*} \pi_{*} \widetilde{E}^{\prime \prime}$ is a bundle on the blowup. But then as in the last paragraph, the canonical sheaf homomorphism $\pi^{*} \pi_{*} \widetilde{E}^{\prime \prime} \rightarrow E^{\prime \prime}$ must be an isomorphism everywhere since the two bundles have the same determinant, contradicting the fact that the former is trivial on $L_{0}$ whereas the latter is not.

The proof of (e) follows from the vanishing of $\pi_{*}^{2} \widetilde{E}(1)$, which implies $\left.\pi_{*}^{1} \widetilde{E} \rightarrow \pi_{*}^{1} \widetilde{E}\right|_{L_{0}}$ is surjective. The proof of (f) will be given later, following the proof of Proposition 2.8 below.

With $\widetilde{E}$ as in Lemma 2.2, suppose that $a_{1} \leq \cdots \leq a_{r}$. The obstruction to extending a section in $\Gamma\left(L_{0}, \widetilde{E}\left(-a_{1}\right)\right)$ to $\widetilde{U}$ lies in $H^{1}\left(\widetilde{U}, \widetilde{E}\left(1-a_{1}\right)\right)$, a group which vanishes by part (d) of the lemma. More generally, any finite number of such sections which are independent over $L_{0}$ will also be so near $L_{0}$. Dualising $\widetilde{E}$, if $\lambda_{1}, \ldots, \lambda_{k} \in \Gamma\left(L_{0}, \widetilde{E}^{*}\left(a_{r}\right)\right)$ are independent, then they can be extended to sections of $\widetilde{E}^{*}\left(a_{r}\right)$ over a neighborhood of $L_{0}$ in $\widetilde{U}$ which are linearly independent at each point in this neighborhood. Rewriting $\left.\widetilde{E}\right|_{L_{0}}$ in the form $\left.\widetilde{E}\right|_{L_{0}}=\oplus_{i=1}^{m} V_{i}\left(b_{i}\right)$ where $V_{i}$ is a vector space with $b_{1}<b_{2}<\ldots<$ $b_{m}$, the last statement implies that the projection $\left.\widetilde{E}\right|_{L_{0}} \rightarrow V\left(b_{m}\right)$ extends to an epimorphism $\widetilde{E} \rightarrow V\left(b_{m}\right)$ in a neighborhood of $L_{0}$. Using induction on rank, this gives the following local description of bundles on the blowup of a two-dimensional ball at a point:

Proposition 2.4. Suppose $\left.\widetilde{E}\right|_{L_{0}}=\oplus_{i=1}^{m} V_{i}\left(b_{i}\right)$ where $V_{i}$ is a $d_{i}$-dimensional vector space and $b_{1}<b_{2}<\cdots<b_{m}$. Then in a neighborhood of $L_{0}$ in $\widetilde{U}$ there is a filtration

$$
0=F_{0} \subset F_{1} \subset \ldots \subset F_{m}=\widetilde{E}
$$

of $\widetilde{E}$ by vector bundles $F_{k}$ such that $F_{k} / F_{k-1} \simeq V_{k}\left(b_{k}\right)$ and such that $\left.F_{k}\right|_{L_{0}}$ $=\bigoplus_{i=1}^{k} V_{i}\left(b_{i}\right)$. 
Corollary 2.6. If $\left.\widetilde{E}\right|_{L_{0}}=\oplus_{i=1}^{r} \mathcal{O}\left(a_{i}\right)$ with $\left|a_{i}-a_{j}\right| \leq 2$ for all $i, j$ then $\widetilde{E} \simeq \oplus_{i=1}^{r} \mathcal{O}\left(a_{i}\right)$ in a neighborhood of $L_{0}$.

Proof. A bundle $F$ on $\widetilde{U}$ which is given as an extension $0 \rightarrow V(a) \rightarrow F \rightarrow$ $W(b) \rightarrow 0$ for some vector spaces $V, W$ is determined by an element of $H^{1}\left(\widetilde{U}, W^{*} \otimes V(a-b)\right)$. If the extension splits on $\pi^{-1}\left(x_{0}\right)$ then this class lies in the image of $H^{1}\left(\widetilde{U}, W^{*} \otimes V(a-b+1)\right) \rightarrow H^{1}\left(\widetilde{U}, W^{*} \otimes V(a-b)\right)$, and if $a-b \geq-2$ then the former group vanishes, by (2.1).

The preceding discussion provides some insight into well-known constructions of Serre [Ser] and of Schwarzenberger [Sch], both described in [OSS]. A bundle $\widetilde{E}$ on $\widetilde{X}$ is the pull-back of a bundle $E$ from $X$ if and only if $\widetilde{E}$ restricts trivially to every component of the exceptional divisor $\widetilde{Y}$; if this is the case, then necessarily $E=\pi_{*} \widetilde{E}$. Let $L_{1}, L_{2}$ be line bundles on $X$ and let $L_{i}$ also denote $\pi^{*} L_{i}$ on $\widetilde{X}$. Extensions of the form $\widetilde{X}: 0 \rightarrow L_{1}(-1) \rightarrow \widetilde{E} \rightarrow L_{2}(1) \rightarrow 0$ are classified by $H^{1}\left(\widetilde{X}, L_{1} L_{2}^{*}(-2)\right)$ which can be computed from the Leray spectral sequence for $\pi$ using (2.1). This gives an exact sequence

$$
\begin{aligned}
0 \rightarrow H^{1}\left(X, L_{1} L_{2}^{*}\right) \rightarrow H^{1}\left(\tilde{X}, L_{1} L_{2}^{*}(-2)\right) & \\
& \rightarrow H^{0}\left(Y, L_{1} L_{2}^{*} \otimes \operatorname{det} \mathcal{N}_{Y}\right) \rightarrow H^{2}\left(X, L_{1} L_{2}^{*}\right)
\end{aligned}
$$

and the bundle $\widetilde{E}$ corresponding to an element of $H^{1}\left(\widetilde{X}, L_{1} L_{2}^{*}(-2)\right)$ is trivial on $\pi^{-1}\left(x_{0}\right)$ if and only if the element of $L_{1} L_{2}^{*} \otimes \operatorname{det} \mathcal{N}_{Y, x_{0}}$ obtained from (2.7) is non-zero. Using (2.1) again, the bundle $E$ is given by an exact sequence $0 \rightarrow L_{1} \rightarrow E \rightarrow L_{2} \otimes \mathcal{I}_{Y} \rightarrow 0$, so $E \otimes L_{1}^{*}$ has a section vanishing precisely at $Y$ and $\mathcal{N}_{Y}$ has been extended to the bundle $E^{*} \otimes L_{2}$. If $X$ is compact, the Chern classes of $E$ are given by $c_{1}(E)=c_{1}\left(L_{1}\right)-c_{1}\left(L_{2}\right)$ and $c_{2}(E)=c_{1}\left(L_{1}\right) c_{1}\left(L_{2}\right)+P D([Y])$ where $P D[Y]$ denotes the Poincaré dual of $[Y]$.

More generally, this construction applies when $Y$ is an arbitrary codimension 2 locally complete intersection in a complex manifold $X$ provided that $\operatorname{det} \mathcal{N}_{Y}$ can be extended to a line bundle on $X$. Indeed, with the exception of Corollary 2.6, all of the results of this section so far presented remain valid if $X$ has arbitrary dimension and $Y$ is a codimension two locally complete intersection; modifications to the proofs above are straightforward.

If $X$ is compact the charge on $\widetilde{E}$ can be estimated in terms of its splitting on $L_{0}=\pi^{-1}\left(x_{0}\right)$ and the charge on its direct image:

Proposition 2.8. Let $\widetilde{X} \stackrel{\pi}{\rightarrow} X$ be the blowup of the compact surface $X$ at $x_{0}$, with $L_{0}=\pi^{-1}\left(x_{0}\right)$. If $\widetilde{E}$ is an r-bundle on $\widetilde{X}$ such that $\left.\widetilde{E}\right|_{L_{0}}=$ 
$\oplus_{i=1}^{r} \mathcal{O}\left(a_{i}\right)$, then for $a:=\sum_{i} a_{i}$ and $E:=\left(\pi_{*} \widetilde{E}\right)^{* *}$ it follows

$$
C(\widetilde{E}) \leq C(E)+\frac{1}{2} \sum_{i=1}^{r}\left(a_{i}-\frac{a}{r}\right)^{2}
$$

with equality iff $\widetilde{E} \simeq \oplus \mathcal{O}\left(a_{i}\right)$ in a neighborhood of $L_{0}$. If $a=0$ then

$$
C(\widetilde{E}) \geq C(E)+\frac{1}{2} \sum_{i=1}^{r}\left|a_{i}\right|
$$

and if $0 \leq a<r$ then

$$
C(\widetilde{E}) \geq C(E)+\frac{a(r-a)}{2 r}+\frac{1}{2} \sum_{a_{i} \leq 0}\left|a_{i}\right|+\frac{1}{2} \sum_{a_{i}>0}\left|a_{i}-1\right|+\frac{1}{2}\left|n_{+}-a\right|,
$$

where $n_{+}:=\#\left\{a_{i} \mid a_{i}>0\right\}$. Hence for any bundle $\widetilde{E}, C(\widetilde{E}) \geq C(E)$ with equality iff $\widetilde{E}=\left(\pi^{*} E\right)(k)$ for some $k \in \mathbb{Z}$.

Remarks. The inequalities (2.9), (2.10) appear in [FM2, Remark 5.4] in the case of rank 2 bundles with $c_{1}=0$.

Note that an arbitrary bundle $\widetilde{E}$ can always be twisted by some $\mathcal{O}(k)$ to ensure that the resulting $a$ satisfies $0 \leq a<r$. Such a twist has no effect on either $C(\widetilde{E})$ or $C(E)$ so $(2.10),(2.11)$ can be used to estimate charges in all cases. Because the invariant form of the formulas are somewhat opaque, these are not given here.

Proof of Proposition 2.8. Since $\chi(\widetilde{E})=\chi\left(\pi_{*} \widetilde{E}\right)-\chi\left(\pi_{*}^{1} \widetilde{E}\right)$ and $c_{1}(\widetilde{X})=$ $\pi^{*} c_{1}(X)+c_{1}(\mathcal{O}(1))$ the Riemann-Roch formula gives

$$
\begin{aligned}
C(\widetilde{E}) & =C\left(\pi_{*} \widetilde{E}\right)+\chi\left(\pi_{*}^{1} \widetilde{E}\right)-\frac{a(a+r)}{2 r} \\
& =C(E)+\operatorname{dim}_{\mathbb{C}}\left(E / \pi_{*} \widetilde{E}\right)+\chi\left(\pi_{*}^{1} \widetilde{E}\right)-\frac{a(a+r)}{2 r} .
\end{aligned}
$$

If $a_{i} \leq 0$ for all $i$ and $\widetilde{E}$ splits in a neighborhood of $L_{0}$ as a direct sum of line bundles then $\pi_{*} \widetilde{E}$ is locally free and $\chi\left(\pi_{*}^{1} \widetilde{E}\right)=-\sum \chi\left(\mathcal{O}\left(a_{i}\right)\right)+$ $\sum \chi\left(\pi_{*} \mathcal{O}\left(a_{i}\right)\right)=(1 / 2) \sum a_{i}\left(a_{i}+1\right)$, giving $C(\widetilde{E})=C(E)+(1 / 2) \sum\left(a_{i}-a / r\right)^{2}$ in this case. If $\widetilde{E}$ does not split in a neighborhood of $L_{0}$ then $\chi\left(\pi_{*}^{1} E\right)$ is strictly less than the corresponding number in the split case - this follows easily by induction on rank using Proposition 2.4. Since (2.9) is invariant under tensoring $\widetilde{E}$ by $\mathcal{O}(-k)$, this proves the upper bound in general.

To prove the lower bounds (2.10) and (2.11), suppose that $0 \leq a<r$ and apply (2.12) to $\widetilde{E}(-1)$ to obtain

$$
C(\widetilde{E})=C(E)+\frac{a(r-a)}{2 r}+\operatorname{dim}_{\mathbb{C}}\left(E / \pi_{*} \widetilde{E}(-1)\right)+\chi\left(\pi_{*}^{1} \widetilde{E}(-1)\right) .
$$


Using Lemma 2.2 as in the proof of Proposition 2.4, in a neighborhood of $L_{0}$ the bundle $\widetilde{E}$ can be written as an extension $0 \rightarrow A \rightarrow \widetilde{E} \rightarrow B \rightarrow 0$ which splits on $L_{0}$ and where $\left.A\right|_{L_{0}}=\oplus_{a_{i} \leq 0} \mathcal{O}\left(a_{i}\right)$ and $\left.B\right|_{L_{0}}=\oplus_{a_{i}>0} \mathcal{O}\left(a_{i}\right)$. Since the extension splits on $L_{0}$, it lies in the image of $H^{1}\left(\widetilde{U}, B^{*} \otimes A(1)\right)$, so there is a bundle $\widetilde{E}_{0}$ on $\widetilde{U}$ which is given by a compatible extension $0 \rightarrow A(1) \rightarrow \widetilde{E}_{0} \rightarrow B \rightarrow 0$. Compatibility of the extension implies that there is an exact sequence $\left.0 \rightarrow \widetilde{E}_{0} \rightarrow \widetilde{E} \rightarrow A\right|_{L_{0}} \rightarrow 0$ giving $\pi_{*} \widetilde{E}_{0}(-1)=\pi_{*} \widetilde{E}(-1)$ and $\left.0 \rightarrow \pi_{*}^{1} \widetilde{E}_{0}(-1) \rightarrow \pi_{*}^{1} \widetilde{E}(-1) \rightarrow \pi_{*}^{1} A(-1)\right|_{L_{0}} \rightarrow 0$, so $\operatorname{dim}_{\mathbb{C}}\left(E / \pi_{*} \widetilde{E}(-1)\right)=$ $\operatorname{dim}_{\mathbb{C}}\left(E / \pi_{*} \widetilde{E}_{0}(-1)\right)$ and $\chi(\widetilde{E}(-1))=\chi\left(\widetilde{E}_{0}(-1)\right)+\sum_{a_{i} \leq 0}\left|a_{i}\right|$. [Although it is not needed in this proof, it is worth pointing out that there is also a map $\widetilde{E} \rightarrow \widetilde{E}_{0}(-1)$ such that the composition $\widetilde{E}_{0} \rightarrow \widetilde{E} \rightarrow \widetilde{E}_{0}(-1)$ is multiplication by a section of $\mathcal{O}(-1)$ defining $L_{0}$.]

It can be assumed without loss of generality that $\widetilde{E}_{0}$ is a bundle on all of $\widetilde{X}$ with $\left(\pi_{*} \widetilde{E}_{0}\right)^{* *}=E$, using the isomorphism $\widetilde{E}_{0} \rightarrow \widetilde{E}$ away from $L_{0}$ to extend the bundle from $\widetilde{U}$ to $\widetilde{X}$; (see below for more on this type of construction). Hence (2.13) applied to $\widetilde{E}(-1)$ gives

$$
C(\widetilde{E})=C\left(\widetilde{E}_{0}\right)+\frac{a(r-a)}{2 r}+\sum_{a_{i} \leq 0}\left|a_{i}\right|+\frac{\left(a-n_{+}\right)\left(a+r-n_{+}\right)}{2 r}
$$

(since $\left.\left.\operatorname{det} \widetilde{E}_{0}\right|_{L_{0}}=\mathcal{O}(a+\operatorname{rank}(A))=\mathcal{O}\left(a+r-n_{+}\right)\right)$. If $n_{+} \leq a$ then $0 \leq\left. c_{1}\left(\widetilde{E}_{0}(-1)\right)\right|_{L_{0}}=a-n_{+}<r$ so $(2.13)$ applied to $\widetilde{E}_{0}(-1)$ gives $C\left(\widetilde{E}_{0}\right)=$ $C\left(\widetilde{E}_{0}(-1)\right) \geq C(E)+\left(a-n_{+}\right)\left(r-a+n_{+}\right) / 2 r$; inserting this in (2.14) gives

$$
C(\widetilde{E}) \geq C(E)+\frac{a(r-a)}{2 r}+\sum_{a_{i} \leq 0}\left|a_{i}\right|+\left(a-n_{+}\right) .
$$

On the other hand, if $n_{+}>a$ then $0 \leq\left. c_{1}\left(\widetilde{E}_{0}\right)\right|_{L_{0}}=a+r-n_{+}<r$, so $C\left(\widetilde{E}_{0}\right) \geq C(E)+\left(a+r-n_{+}\right)\left(n_{+}-a\right) / 2 r$ which implies

$$
C(\widetilde{E}) \geq C(E)+\frac{a(r-a)}{2 r}+\sum_{a_{i} \leq 0}\left|a_{i}\right|
$$

in this case. Thus in either case,

$$
C(\widetilde{E}) \geq C(E)+\frac{a(r-a)}{2 r}+\sum_{a_{i} \leq 0}\left|a_{i}\right|+\max \left\{a-n_{+}, 0\right\} .
$$

If $a=0$ replace $\widetilde{E}$ by $\widetilde{E}^{*}$ to obtain the lower bound $C(\widetilde{E}) \geq C(E)+$ $\sum_{a_{i} \geq 0}\left|a_{i}\right|$; averaging the two bounds gives $(2.10)$. If $0<a<r$ replace $\widetilde{E}$ by $\widetilde{E}^{*}(1)$ to obtain the lower bound $C(\widetilde{E}) \geq C(E)+\sum_{a_{i} \geq 1}\left|1-a_{i}\right|+a(r-$ a) $/ 2 r+\max \left\{n_{+}-a, 0\right\}$; averaging this with (2.15) yields (2.11).

Clearly $C(\widetilde{E})=C(E)$ implies all $a_{i}$ must be equal to 0 , so $\widetilde{E}=\pi^{*} E$ by Lemma 2.2 in this case. 
The lower bounds in (2.10) and (2.11) are sharp: Given any splitting type $\oplus \mathcal{O}\left(a_{i}\right)$ for a bundle $\widetilde{E}$ on $L_{0}$, such that $a \leq n_{+}$there is an extension of the form $0 \rightarrow A(1) \rightarrow \widetilde{E}_{0} \rightarrow B \rightarrow 0$ with $A$ and $B$ of the above form and with $\widetilde{E}_{0}$ a direct sum of the line bundles $\mathcal{O}$ and $\mathcal{O}(1)$. If $a>n_{+}$, replacing $a_{i}$ by $1-a_{i}$ for all $i$ (which has the effect of replacing $\widetilde{E}$ by $\widetilde{E}^{*}(1)$ ) ensures that this criterion is met.

Proof of Lemma 2.2(f). Let $\widetilde{E}$ be an $r$-bundle on $\widetilde{U}$ with $\pi_{*} \widetilde{E}(-1)$ locally free and $\pi_{*}^{1} \widetilde{E}(-1)=0$; it will be shown that $\widetilde{E}(-1)$ is a direct sum of the line bundles $\mathcal{O}(-1)$ and $\mathcal{O}(0)$.

As in the proof of Proposition 2.8 it can be supposed that $\widetilde{E}$ is a bundle on $\widetilde{\mathbb{P}}_{2}$ such that $\left(\pi_{*} \widetilde{E}\right)^{* *}$ is trivial; (for more details see below). From (2.12) applied to $\widetilde{E}(-1)$ it follows $C(\widetilde{E})=a(r-a) / 2 r$ where $\left.c_{1}(\widetilde{E})\right|_{L_{0}}=a$. Since $C(\widetilde{E}) \geq 0$ it follows that $0 \leq a \leq r$, and $a=r$ implies $\widetilde{E}=\oplus_{i=1}^{r} \mathcal{O}(1)$. Assuming therefore without loss of generality that $a<r$, it follows from (2.11) that $\widetilde{E}$ splits on $L_{0}$ as a direct sum of the line bundles $\mathcal{O}(0)$ and $\mathcal{O}(1)$, and therefore it splits in this way in a neighborhood of $L_{0}$, by Corollary 2.6.

Remark. If $\mathcal{S}$ is a torsion-free sheaf such that $\pi_{*}^{1} \mathcal{S}=0$, a simple diagram chase yields the exact sequence $0 \rightarrow \pi_{*}\left(\mathcal{S}^{* *} / \mathcal{S}\right) \rightarrow\left(\pi_{*} \mathcal{S}\right)^{* *} / \pi_{*} \mathcal{S} \rightarrow$ $\left(\pi_{*} \mathcal{S}^{* *}\right)^{* *} / \pi_{*} \mathcal{S}^{* *} \rightarrow 0$. Thus if in addition $\pi_{*} \mathcal{S}$ is locally free, it follows that $\mathcal{S}$ is in fact locally free and splits as a sum line bundle of the form $\mathcal{O}$ and $\mathcal{O}(-1)$ in $\widetilde{U}$.

As before, let $X$ be a complex surface and let $\widetilde{X} \stackrel{\pi}{\rightarrow} X$ be the blowup of $X$ at the point $x_{0} \in X$. The local description provided by Proposition 2.4 can be combined with a holomorphic version of Taubes' "cut-and-paste" construction $[\mathbf{T}]$ to provide a global description of bundles on $\widetilde{X}$.

Let $\widetilde{E}$ be a holomorphic $r$-bundle on $\widetilde{X}$, and let $U \subset X$ be a neighborhood of $x_{0}$ isomorphic to a ball. Then $\widetilde{E}$ can be viewed as comprised of two pieces, namely the bundle $\left(\pi_{*} \widetilde{E}\right)^{* *}$ on $X$ and the bundle $\left.\widetilde{E}\right|_{\widetilde{U}}$ on $\widetilde{U}=\pi^{-1}(U)$; the two pieces are glued together by means of the isomorphism $\pi_{*} \widetilde{E} \simeq\left(\pi_{*} \widetilde{E}\right)^{* *}$ over $U \backslash\left\{x_{0}\right\} \simeq \widetilde{U} \backslash L$.

Conversely, given $r$-bundles $E_{0}$ on $X$ and $E_{1}$ on $\widetilde{U}$, the two can be glued together by means of an isomorphism $\rho: \pi_{*} E_{1} \rightarrow E_{0}$ over $U \backslash\left\{x_{0}\right\}$, extending uniquely to $U$ as an isomorphism $\left.\left(\pi_{*} E_{1}\right)^{* *} \rightarrow E_{0}\right|_{U}$ to define a bundle $E_{0} \#_{\rho} E_{1}$ on $\tilde{X}$. If $\left(E_{0}^{\prime}, E_{1}^{\prime}, \rho^{\prime}\right)$ is another triple of such objects such that $E_{0} \#{ }_{\rho} E_{1} \leadsto E_{0}^{\prime} \# \rho^{\prime} E_{1}^{\prime}$, then by Hartogs' theorem the isomorphism $\left.E_{0}\right|_{X \backslash \bar{U}}$ $\left.\leadsto E_{0}^{\prime}\right|_{X \backslash \bar{U}}$ extends to an isomorphism $\phi_{0}$ over $X$, and if $\phi_{1}$ denotes the induced isomorphism $E_{1} \rightarrow E_{1}^{\prime}$, it follows that $\rho^{\prime}=\phi_{0} \rho \pi_{*} \phi_{1}^{-1}$. 
If $\rho_{0}:\left.\left(\pi_{*} E_{1}\right)^{* *} \rightarrow E_{0}\right|_{U}$ is fixed and $\rho$ is any other such isomorphism, then $\rho \rho_{0}^{-1}$ is an automorphism of $E_{0}$ over $U$. Hence the following description is obtained:

Proposition 2.16. Let $E_{0}$ be a bundle on $X, E_{1}$ be a bundle on a neighborhood $\pi^{-1}(U)$ of $\pi^{-1}\left(x_{0}\right)$ in the blowup $\widetilde{X}$ of $X$ at $x_{0}$ and $\rho_{0}:\left.\left(\pi_{*} E_{1}\right)^{* *} \simeq E_{0}\right|_{U}$ be given. Then isomorphism classes of vector bundles $\widetilde{E}$ on $\widetilde{X}$ such that $\left(\pi_{*} \widetilde{E}\right)^{* *} \simeq E_{0}$ and $\widetilde{E} \simeq E_{1}$ in a neighborhood of $\pi^{-1}\left(x_{0}\right)$ are parameterised by the stalk of the skyscraper sheaf $\mathcal{A} u t\left(E_{0}\right) / \rho_{0} \pi_{*} \mathcal{A} u t\left(E_{1}\right) \rho_{0}^{-1}$ at $x_{0}$, modulo the left action of $\Gamma\left(X, \operatorname{Aut}\left(E_{0}\right)\right)$.

\section{Remarks.}

1. If $E_{1}=\bigoplus \mathcal{O}\left(a_{i}\right)$, the space $\mathcal{A} u t\left(\left(\pi_{*} E_{1}\right)^{* *}\right) / \pi_{*} \mathcal{A} u t\left(E_{1}\right)$ is easily identified with the total space of some homogeneous vector bundle over a flag manifold. Two simple examples which will be of some relevance subsequently are the cases $E_{1}=\mathcal{O}(1) \oplus \mathcal{O}^{r-1}$ and $E_{1}=\mathcal{O}(-1) \oplus \mathcal{O}(1) \oplus \mathcal{O}^{r-2}$, for which the corresponding skyscrapers are respectively $\mathbb{F}_{1}\left(\mathbb{C}^{r}\right)$ and the total space of the bundle $2 \mathcal{O}(1,1)$ over $\mathbb{F}_{1, r-1}\left(\mathbb{C}^{r}\right)$; (the zero section corresponds to those bundles which extend to $\widetilde{\mathbb{P}}_{2}$ as a direct sum of line bundles).

2. This description provides a simple way to construct bundles on $\tilde{X}$ from bundles on $X$, but in contrast with the construction of Serre/Schwarzenberger the bundles produced this way are all non-trivial on the exceptional divisor. However, if $\sum_{i} a_{i}=0$ the generic deformation of the bundle $\widetilde{E}$ (or $E_{1}$ ) will be trivial on $L$, and the earlier construction can be seen as a deformation of a bundle restricting to $\mathcal{O}(-1) \oplus \mathcal{O}(1)$ on $L$; (the existence of such deformations is discussed further in $\S 4$ ).

An effective description of the spaces of holomorphic bundles on $\widetilde{X}$ requires such a description for the spaces of bundles in a neighborhood of $L_{0}$, but that given by Proposition 2.4 has some redundancy: The filtration (2.5) is not uniquely determined. However, by gluing a bundle on a neighborhood of $L_{0}$ to the trivial bundle on $\mathbb{P}_{2}$ the classification problem becomes that of determining the bundles on $\widetilde{\mathbb{P}}_{2}$ which are trivial in a neighborhood of the line $L_{\infty}$ at infinity. A trivialisation of such a bundle in a neighborhood of $L_{\infty}$ is determined by its restriction to $L_{\infty}$, so isomorphism classes of pairs $\left(E_{1}, \rho\right)$ where $\rho$ is a trivialisation of $\left(\pi_{*} E_{1}\right)^{* *}$ in a neighborhood of $x_{0}$ correspond to isomorphism classes of pairs $(\widetilde{E}, \varphi)$ where $\widetilde{E}$ is a bundle on $\widetilde{\mathbb{P}}_{2}$ such that $\left(\pi_{*} \widetilde{E}\right)^{* *}$ is trivial and $\varphi$ is a trivialisation of $\widetilde{E}$ on $L_{\infty}$.

The space $\widetilde{\mathbb{P}}_{2}$ is isomorphic to the Hirzebruch surface $H_{1}$ and bundles on this space have been studied in [B2]. Using the lemma of $\S 1$ of that reference, a monad description of all holomorphic bundles on $H_{1}$ trivial on $L_{\infty}$ is easily given, and the precise condition on such monads for the 
corresponding bundles to be trivial in a neighborhood of $L_{\infty}$ is quite easily calculated.

\section{Structure of moduli spaces I.}

The "cut-and-paste" construction of the previous section makes no reference to questions of stability, an issue which is considered in this section. When the discussion is not limited to a single bundle but rather to whole moduli spaces, the results generally apply only in the case that $b_{1}(X)$ is even; the reason for this can be traced to the failure of Lemma 1.8 when $b_{1}(X)$ is odd.

The definition of stability requires a hermitian metric, and throughout this section such a metric (positive $(1,1)$-form) $\omega$ is a fixed on $X$. The metrics to be used on blowups of $X$ are the same as those used in [B3], the construction of which will be briefly recalled here for convenience.

Let $\tilde{X} \stackrel{\pi}{\rightarrow} X$ be the blowup of $X$ at $x_{0} \in X$. Let $L:=\pi^{-1}\left(x_{0}\right)$ be the exceptional divisor so $\pi^{*} \omega$ is everywhere non-negative and is degenerate only in directions tangent to $L$. Let $\sigma$ be $i / 2 \pi$ times the curvature form of any hermitian connection on the line bundle $\mathcal{O}(-L)=: \mathcal{O}(1)$ restricting positively to $L$, and let $\omega_{\epsilon}:=\pi^{*} \omega+\epsilon \sigma$ for $\epsilon>0$; (recall $\left.\mathcal{O}(L)\right|_{L} \simeq \mathcal{O}_{L}(-1)$ ). It follows that if $\epsilon$ is sufficiently small then $\omega_{\epsilon}$ defines a positive form in a neighborhood of $L$; if $\sigma$ is compactly supported in $\widetilde{X}$ then $\omega_{\epsilon}$ is everywhere positive for sufficiently small $\epsilon$. If $\omega$ is $\bar{\partial} \partial$-closed and $\sigma$ is compactly supported, it follows from the fact that $L$ has self-intersection -1 that $\operatorname{Vol}\left(\tilde{X}, \omega_{\epsilon}\right)=\operatorname{Vol}(X, \omega)-\epsilon^{2} / 2=V-\epsilon^{2} / 2$, and if $\omega$ is $d$-closed, then so too is $\omega_{\epsilon}$.

A useful model to keep in mind is the following: If $x_{0}$ corresponds to the origin in local holomorphic coordinates $\left\{z^{a}\right\}$, the orientation-reversing map $z^{a} \mapsto z^{a} /|z|^{2}$ lifts to the blowup to define an isomorphism of a neighborhood of $L$ with a neighborhood of a line in $\mathbb{C P}_{2}$, realising $\widetilde{X}$ as the connected sum $\widetilde{X} \simeq_{\text {diffeo }} X \# \overline{\mathbb{C P}}_{2}$. Under this diffeomorphism, the pull-back of the Riemannian metric corresponding to $\omega_{1}=(i / 2) \partial \bar{\partial}\left(|z|^{2}+\log |z|^{2}\right)$ is conformal to the Fubini-Study metric. The form $\sigma$ can be taken to be $(i / 2) \partial \bar{\partial} \log \left(\psi\left(|z|^{2}\right)\right)$ where $\psi(t)$ is a smooth function which is the identity near 0 and a positive constant for $t \geq t_{0}$. Pulling back under the "dilations" $z \mapsto \epsilon^{-1 / 2} z$ and rescaling by $\epsilon$ gives the metric $\omega_{\epsilon}$, "stretching out" the neck of the connected sum as in [D3].

Now let $\widetilde{X} \stackrel{\pi}{\rightarrow} X$ be a modification of $X$ consisting of $n$ successive blowups, and let $\sigma_{i}$ be a closed smooth $(1,1)$-form on $\widetilde{X}$ corresponding as in the last paragraph to the $i$-th blowup. Let $\mathbb{R}_{+}^{n}:=\left\{\alpha=\left(\alpha_{1}, \ldots, \alpha_{n}\right) \in\right.$ $\left.\mathbb{R}^{n} \mid \alpha_{i}>0, i=1, \ldots, n\right\}$ and for $\alpha \in \mathbb{R}_{+}^{n}$ let $\rho_{\alpha}:=\sum \alpha_{i} \sigma_{i}$, so $\rho_{\alpha} \cdot \rho_{\alpha}=$ $-\sum \alpha_{i}^{2}=:-|\alpha|^{2}$; (this definition differs slightly from that in [B3] where $\rho$ has the opposite sign). If there are no multiple blowups, $\omega_{\alpha}:=\pi^{*} \omega+\rho_{\alpha}$ 
is positive for all $\alpha \in \mathbb{R}_{+}^{n}$ with $|\alpha|$ sufficiently small; if there are multiple blowups then not all such $\alpha$ can define a positive form. A vector $\alpha \in \mathbb{R}_{+}^{n}$ is called suitable if $\omega_{\alpha}$ is a positive form on $\widetilde{X}$.

Let $\tilde{X} \stackrel{\pi}{\rightarrow} X$ be a blowup of the compact surface $X$, equipped with a metric of the form $\omega_{\alpha}$ as above. Let $\widetilde{E}$ be a holomorphic bundle on $\widetilde{X}$, and let $\widetilde{A} \subset \widetilde{E}$ be a bundle of rank $a$ included in $\widetilde{E}$ as a subsheaf. By definition of $\nu_{\bullet}(*)$,

$$
\nu_{\widetilde{E}}\left(\widetilde{A}, \omega_{\alpha}\right)=\nu_{\pi_{*} \widetilde{E}}\left(\pi_{*} \widetilde{A}\right)+\rho_{\alpha} \cdot\left[\frac{a}{r} c_{1}(\widetilde{E})-c_{1}(\widetilde{A})\right] .
$$

If $\widetilde{A}$ has torsion-free quotient $\widetilde{C}$ of rank $c>0$ then (1.2) and (1.4) give

$$
\begin{aligned}
C(\widetilde{E})=C(\widetilde{A})+C(\widetilde{C})+\frac{r}{2 a c} & {\left[\left\|\frac{a}{r} c_{1}\left(\pi_{*} \widetilde{E}\right)-c_{1}\left(\pi_{*} \widetilde{A}\right)\right\|_{\omega}^{2}\right.} \\
+ & \left.\left\|\frac{a}{r} c_{1}(\widetilde{E})-c_{1}(\widetilde{A})\right\|_{Q}^{2}-\frac{\nu_{\pi_{*} \widetilde{E}}\left(\pi_{*} \widetilde{A}\right)^{2}}{V}\right],
\end{aligned}
$$

where $\|x\|_{Q}^{2}:=-\left(x-\pi^{*} \pi_{*} x\right) \cdot\left(x-\pi^{*} \pi_{*} x\right)$ for $x \in H^{2}(\widetilde{X}, \mathbb{Q})$.

Suppose now that $\widetilde{E}$ is semi-stable but not stable with respect to $\omega_{\alpha}$, and that $\widetilde{A}$ destabilizes $\widetilde{E}$. Then from (3.1) it follows that $\nu_{\pi_{*} \widetilde{E}}\left(\pi_{*} \widetilde{A}\right)=$ $-\rho_{\alpha} \cdot\left[(a / r) c_{1}(\widetilde{E})-c_{1}(\widetilde{A})\right]$ so $(3.2)$ implies

$$
\begin{aligned}
C(\widetilde{E}) \geq C(\widetilde{A})+C(\widetilde{C})+\frac{r}{2 a c}[ & \left\|\frac{a}{r} c_{1}\left(\pi_{*} \widetilde{E}\right)-c_{1}\left(\pi_{*} \widetilde{A}\right)\right\|_{\omega}^{2} \\
& \left.+\left(1-\frac{|\alpha|^{2}}{V}\right)\left\|\frac{a}{r} c_{1}(\widetilde{E})-c_{1}(\widetilde{A})\right\|_{Q}^{2}\right]
\end{aligned}
$$

Since $\widetilde{A}$ destabilizes $\widetilde{E}$ for the metric $\omega_{\alpha}$, it follows from the semi-stability of $\widetilde{E}$ that both $\widetilde{A}$ and $\widetilde{C}$ are also semi-stable (with respect to this metric), implying $C(\widetilde{A}), C(\widetilde{C})$ are non-negative. Hence (3.3) yields a uniform bound on $\left\|(a / r) c_{1}(\widetilde{E})-c_{1}(\widetilde{A})\right\|$ which involves only $C(\widetilde{E})$ and $r$ (if $|\alpha|$ is suitably bounded from above).

With these preparations in hand, the following result summarises most of the important relationships between stability on $X$ and $\widetilde{X}$. Parts of it appear in [FM2, Theorem 5.5] and [Br, Theorem 4] in the case of bundles of rank 2 .

Proposition 3.4. Let $\widetilde{E}$ be an r-bundle on $\widetilde{X}$.

(a) If $\widetilde{E}$ is $\omega_{\epsilon \alpha}$-stable for all sufficiently small $\epsilon>0$ then $\pi_{*} \widetilde{E}$ is $\omega$-semistable;

(b) If $\widetilde{E}=\pi^{*} E$ for some bundle $E$ on $X$ and $\widetilde{E}$ is $\omega_{\alpha}$-stable, then $E$ is $\omega$-stable; 
(c) If $\widetilde{E}$ is $\omega_{\alpha}$-(semi-) stable and $\pi_{*} \widetilde{E}$ is $\omega$-semi-stable, then $\widetilde{E}$ is $\omega_{\epsilon \alpha^{-}}$ (semi-) stable for all $\epsilon \in(0,1]$;

(d) If $\pi_{*} \widetilde{E}$ is stable, then $\widetilde{E}$ is $\omega_{\alpha}$-stable for all suitable $\alpha \in \mathbb{R}_{+}^{n}$ sufficiently small. In fact, if $\nu_{\pi_{*} \widetilde{E}}(A) \geq \delta>0$ for all $A \subset \pi_{*} \widetilde{E}$ with non-zero torsion-free quotient, then $\widetilde{E}$ is $\omega_{\alpha}$-stable for all suitable $\alpha \in \mathbb{R}_{+}^{n}$ such that $|\alpha|<\delta \sqrt{2 V /\left(2 \delta^{2}+r V C(\widetilde{E})\right)}$.

Proof. (a) By Lemma 2.2, after twisting $\widetilde{E}$ with a suitable line bundle it can be supposed that $\pi_{*} \widetilde{E}$ is locally free. If $A \subset \pi_{*} \widetilde{E}$ has torsion-free quotient, then $\pi^{*} A \subset \pi^{*} \pi_{*} \widetilde{E} \subset \widetilde{E}$. If $\widetilde{A}$ is the maximal normal extension of $\pi^{*} A$ in $\widetilde{E}$ then $\mu\left(\pi^{*} A, \omega_{\alpha}\right) \leq \mu\left(\widetilde{A}, \omega_{\alpha}\right)$. Replacing $\alpha$ by $\epsilon \alpha$ in (3.1) and letting $\epsilon \rightarrow 0$ gives $\nu_{\pi_{*} \widetilde{E}}(A) \geq 0$.

(b) If $A \subset E$ has torsion-free quotient and $\widetilde{A} \subset \widetilde{E}$ is the maximal normal extension of $\pi^{*} A$ in $\widetilde{E}=\pi^{*} E$, then $\mu(A, \omega)=\mu\left(\pi^{*} A, \omega_{\alpha}\right) \leq \mu\left(\widetilde{A}, \omega_{\alpha}\right)<$ $\mu\left(\widetilde{E}, \omega_{\alpha}\right)=\mu(E, \omega)$.

(c) Suppose $\widetilde{A} \subset \widetilde{E}$ has torsion-free quotient. If $\nu_{\widetilde{E}}\left(\widetilde{A}, \omega_{\epsilon \alpha}\right)<0$ for some $\epsilon \in(0,1)$ then since $\nu_{\pi_{*} \widetilde{E}}\left(\pi_{*} \widetilde{A}\right) \geq 0$ by hypothesis it would follow that $\epsilon \rho_{\alpha}$. $\left[(a / r) c_{1}(\widetilde{E})-c_{1}(\widetilde{A})\right]<0$; this would imply $\nu_{\widetilde{E}}\left(\widetilde{A}, \omega_{\alpha}\right)<0$ also, contradicting the hypotheses. If $\widetilde{A}$ destabilizes $\widetilde{E}$ for $\omega_{\epsilon \alpha}$ then both $\nu_{\pi_{*} \widetilde{E}}\left(\pi_{*} \widetilde{A}\right)$ and $\rho_{\alpha}$. $\left[(a / r) c_{1}(\widetilde{E})-c_{1}(\widetilde{A})\right]$ must be zero. Otherwise, one must be strictly positive and therefore $\widetilde{E}$ must be strictly stable with respect to $\omega_{\epsilon \alpha}$ for all $\epsilon \in(0,1]$. (d) If $\widetilde{A} \subset \widetilde{E}$ has torsion-free quotient, the first Chern class of $\widetilde{A}$ restricted to any irreducible component of the exceptional divisor is bounded above by a constant depending on the maximum of the first Chern classes of the line bundles in the decomposition of $\widetilde{E}$ on that component. The proof of Lemma 5 of [B3] now applies to show that if $\widetilde{E}$ has at least one non-trivial subsheaf, then there exists such a subsheaf $\widetilde{A}$ with torsion-free quotient $\widetilde{C}$ which maximizes $\mu\left(-, \omega_{\epsilon \alpha}\right)$ for any sufficiently small $\epsilon>0$. It then follows from (3.1) that $\widetilde{E}$ is stable with respect to $\omega_{\epsilon \alpha}$ for all $\epsilon$ sufficiently small.

If $\alpha$ is suitable and satisfies the inequality of (d) and $\widetilde{E}$ is not $\omega_{\alpha}$-stable, then since stability is an open condition on the metric there exists $\epsilon \in(0,1]$ such that $\widetilde{E}$ is $\omega_{\epsilon \alpha}$-semi-stable but not stable. Then $\delta \leq \nu_{\pi_{*} \widetilde{E}}(\widetilde{A})=-\epsilon \rho_{\alpha}$. $\left((a / r) c_{1}(\widetilde{E})-c_{1}(\widetilde{A})\right) \leq|\alpha|\left\|c_{1}(\widetilde{E})-c_{1}(\widetilde{A})\right\|_{Q}$. Replacing $\alpha$ by $\epsilon \alpha$ in (3.3) gives the bound $\left\|c_{1}(\widetilde{E})-c_{1}(\widetilde{A})\right\|_{Q}^{2} \leq r V C(\widetilde{E}) /\left(2 V-2 \epsilon^{2}|\alpha|^{2}\right)$, which gives the contradiction $\delta<\delta$ after a little algebra.

A simple corollary of the proposition is that if $\widetilde{E}=E_{0} \#_{\rho} E_{1}$ is a bundle on $\widetilde{X}$ constructed by the gluing construction of the last section and if $E_{0}$ 
on $X$ is $\omega$-stable, then $\widetilde{E}$ is $\omega_{\alpha}$-stable for all suitable $\alpha \in \mathbb{R}_{+}^{n}$ sufficiently small. The gluing construction, combined with the existence of HermitianEinstein connections on stable bundles, can thus be viewed as a holomorphic interpretation of Donaldson's "connected sums of connections" theorem [D3] where one of the summands is (a connected sum of copies of) $\overline{\mathbb{P}}_{2}$.

By Lemma 1.8, Proposition 2.8 and part (d) of Proposition 3.4, when $b_{1}(X)$ is even it follows that for any bundle $\widetilde{E}$ on $\widetilde{X}$ of rank $r$ and charge $\leq C_{0}$ such that $\pi_{*} \widetilde{E}$ is stable, $\widetilde{E}$ is $\omega_{\alpha}$-stable for all suitable $\alpha \in \mathbb{R}_{+}^{n}$ satisfying a uniform bound independent of $\widetilde{E}$. In particular, the pull-backs from $X$ of stable bundles of bounded ranks and charge are all stable with respect to the same metrics on the blowup. The restriction that $b_{1}(X)$ be even gives the following strengthening of Proposition 3.4:

Proposition 3.5. Suppose that $b_{1}(X)$ is even. For any $r_{0}, C_{0}>0$ there exists $\epsilon_{0}=\epsilon_{0}\left(r_{0}, C_{0}, \omega\right)$ with the property that any bundle on a blowup $\widetilde{X}$ of $X$ of rank $\leq r_{0}$ and charge $\leq C_{0}$ which is stable with respect to $\omega_{\alpha}$ for some suitable $\alpha \in \mathbb{R}_{+}^{n}$ satisfying $\left|\alpha_{0}\right|<\epsilon_{0}$ is stable with respect to $\omega_{\epsilon \alpha}$ for all $\epsilon \in\left(0, \epsilon_{0} /|\alpha|\right)$. Moreover, any bundle which is semi-stable with respect to $\omega_{\alpha}$ and has semi-stable direct image is semi-stable with respect to $\omega_{\epsilon \alpha}$ for all $\epsilon \in\left(0, \epsilon_{0} /|\alpha|\right)$.

Proof. Set $\epsilon_{1}:=\delta_{0} \sqrt{2 V /\left(2 \delta_{0}^{2}+r V C(\widetilde{E})\right)}$ where $\delta_{0}$ is as in Lemma 1.8. Suppose that there exists suitable $\alpha_{1} \in \mathbb{R}_{+}^{n}$ with $\left|\alpha_{1}\right|<\epsilon_{1}$ and a bundle $\widetilde{E}_{1}$ of rank $r_{1} \leq r_{0}$ on $\widetilde{X}$ with $C\left(\widetilde{E}_{1}\right) \leq C_{0}$ which is $\omega_{\alpha_{1}}$-stable but which is not $\omega_{\delta_{1} \alpha_{1}}$-stable for some $\delta_{1} \in\left(0, \epsilon_{1} /\left|\alpha_{1}\right|\right)$, where $\delta_{1} \alpha_{1}$ is suitable. Since stability is an open condition on the metric, by altering $\delta_{1}$ if necessary it can be supposed that $\widetilde{E}_{1}$ is semi-stable but not stable with respect to $\omega_{\delta_{1} \alpha}$, and hence that there exists $\widetilde{A}_{1} \subset \widetilde{E}_{1}$ destabilizing as in the discussion preceding Proposition 3.4. Set $\epsilon_{2}:=(1 / 2) \min \left\{\left|\alpha_{1}\right|, \delta_{1}\left|\alpha_{1}\right|\right\}$ and repeat this procedure, generating a sequence $\left\{\left(\epsilon_{i}, \alpha_{i}, \widetilde{E}_{i}, \widetilde{A}_{i}, \delta_{1}\right)\right\}$ by iteration. By construction,

$$
\begin{array}{r}
\nu_{\pi_{*} \widetilde{E}_{i}}\left(\pi_{*} \widetilde{A}_{i}\right)+\rho_{\alpha_{i}} \cdot\left[\left(a_{i} / r_{i}\right) c_{1}\left(\widetilde{E}_{i}\right)-c_{1}\left(\widetilde{A}_{i}\right)\right]>0 \\
\nu_{\pi_{*} \widetilde{E}_{i}}\left(\pi_{*} \widetilde{A}_{i}\right)+\delta_{i} \rho_{\alpha_{i}} \cdot\left[\left(a_{i} / r_{i}\right) c_{1}\left(\widetilde{E}_{i}\right)-c_{1}\left(\widetilde{A}_{i}\right)\right]=0,
\end{array}
$$

with $\delta_{1}\left|\alpha_{i}\right|<\epsilon_{i}<\epsilon_{i-1} / 2$. Since $1 \leq a_{i} \leq r_{i} \leq r_{0}$ and $C\left(\widetilde{E}_{i}\right) \leq C_{0}$ the uniform bound on the norms $\left\|\left(a_{i} / r_{i}\right) c_{1}\left(\widetilde{E}_{i}\right)-c_{1}\left(\widetilde{A}_{i}\right)\right\|$ provided by (3.3) implies that there is a subsequence for which $\left(a_{i} / r_{i}\right) c_{1}\left(\widetilde{E}_{i}\right)-c_{1}\left(\widetilde{A}_{i}\right)$ is constant. Since $b_{1}(X)$ is even, $\nu_{\bullet}(*)$ is topological and therefore constant on this subsequence. If the subsequence is infinite, then $\epsilon_{i} \rightarrow 0$, so (3.6)(b) implies $\nu_{\pi_{*} \widetilde{E}_{i}}\left(\pi_{*} \widetilde{A}_{i}\right)$ is eventually 0 on this subsequence and therefore so too is 
$\rho_{\alpha_{i}} \cdot\left[\left(a_{i} / r_{i}\right) c_{1}\left(\widetilde{E}_{i}\right)-c_{1}\left(\widetilde{A}_{i}\right)\right]$; this however contradicts $(3.6)(\mathrm{a})$. Thus the subsequence must be finite, implying the original sequence terminated, which in turn implies the first statement of the proposition.

To prove the second statement, suppose that $\widetilde{E}$ is $\omega_{\alpha}$-semi-stable for some suitable $\alpha \in \mathbb{R}_{+}^{n}$ with $|\alpha|<\epsilon_{0}$, and that $\pi_{*} \widetilde{E}$ is semi-stable. If $\widetilde{E}$ is $\omega_{\alpha^{-}}$ stable then the first part of the proposition applies, so it can be assumed that $\widetilde{E}$ is not stable. If $\widetilde{A} \subset \widetilde{E}$ has torsion-free quotient and destabilizes $\widetilde{E}$ with respect to $\omega_{\alpha}$, then $\nu_{\pi_{*} \widetilde{E}}\left(\pi_{*} \widetilde{A}\right)=-\rho_{\alpha} \cdot\left[(a / r) c_{1}(\widetilde{E})-c_{1}(\widetilde{A})\right] \leq$ $|\alpha|\left\|(a / r) c_{1}(\widetilde{E})-c_{1}(\widetilde{A})\right\|_{Q}<\delta_{0}$ by construction of $\epsilon_{0}$ and by $(3.3)$, so by Lemma 1.8 it follows $\nu_{\pi_{*} \widetilde{E}}\left(\pi_{*} \widetilde{A}\right)=0=\rho_{\alpha} \cdot\left[(a / r) c_{1}(\widetilde{E})-c_{1}(\widetilde{A})\right]$. Hence $\nu_{\widetilde{E}}\left(\widetilde{A}, \omega_{\epsilon \alpha}\right)=0$ for all $\epsilon$.

In general, it is not the case that the moduli spaces of $\omega_{\alpha}$-stable holomorphic structures on a given topological bundle over $\widetilde{X}$ are independent of $\alpha \in \mathbb{R}_{+}^{n}$ once $|\alpha|$ is sufficiently small. If $\widetilde{E}$ is a bundle on $\widetilde{X}$ of rank $\leq r_{0}$ and charge $\leq C_{0}$ which is stable with respect to $\omega_{\alpha}$ but not stable with respect to $\omega_{\beta}$ for $|\alpha|,|\beta| \leq \epsilon_{0}$, then it follows easily as in the proof of Proposition 3.5 that there exists $c \in H^{2}(X, \mathbb{Z})^{\perp} \subset H^{2}(\widetilde{X}, \mathbb{Z}) \cap H^{1,1}(\widetilde{X})$ with $\|c\|_{Q} \leq \sqrt{r_{0}^{3} V C_{0} /\left(2 V-2 \epsilon_{0}^{2}\right)}$ such that $\rho_{\alpha} \cdot c>0$ and $\rho_{\beta} \cdot c \leq 0$, namely $c=\left(a c_{1}(\widetilde{E})-r c_{1}(\widetilde{A})\right)-\left(a \pi^{*} c_{1}\left(\pi_{*} \widetilde{E}\right)-r \pi^{*} c_{1}\left(\pi_{*} \widetilde{A}\right)\right)$ for some destabilizing $\widetilde{A} \subset \widetilde{E}$. The moduli spaces will be independent of suitable $\alpha \in \mathbb{R}_{+}^{n}$ satisfying $|\alpha|<\epsilon_{0}$ provided that $\alpha$ remains within one of the finitely many chambers of $\mathbb{R}_{+}^{n}$ cut out by the equations $\rho_{\alpha} \cdot c=0$ for $c \in H^{2}(X, \mathbb{Z})^{\perp}$ with $\|c\|_{Q} \leq \sqrt{r_{0}^{3} V C_{0} /\left(2 V-2 \epsilon_{0}^{2}\right)}$. Such a "chamber structure" for moduli spaces is quite well-known - see, e.g., [D4], [K2].

Moduli spaces also depend non-trivially on $|\alpha|$ in general: It is not hard to construct examples of bundles on a blowup $\widetilde{X}$ which are stable with respect to $\omega_{\alpha}$ for some suitable $\alpha$, but which fail to be stable with respect to $\omega_{\epsilon \alpha}$ for some $\epsilon \in(0,1)$.

\section{Stabilization and desingularisation.}

The appearance of sheaves and bundles which are semi-stable but not stable represents a divergence between the real analytical and the complex analytical descriptions of moduli: Whereas isomorphism classes of stable bundles and irreducible Hermitian-Einstein connections are in one-to-one correspondence, this fails to be true as soon as stable is replaced by semi-stable: For example, if $A$ and $B$ are stable bundles with $\mu(A)=\mu(B)$, any extension of the form $0 \rightarrow A \rightarrow E \rightarrow B \rightarrow 0$ defines a bundle $E$ with $\mu(E)=\mu(B)$ which is always semi-stable but not stable. The bundle $E$ admits a HermitianEinstein connection if and only if the extension splits. In this section, the 
gluing construction of $\S 2$ is used to provide a mechanism for "stabilizing" a semi-stable bundle (or torsion-free sheaf). The methods, which are strictly sheaf-theoretical, can also be used to by-pass some of the technical difficulties encountered in [D5] to show that moduli spaces of stable bundles of sufficiently large charge on a blowup of a surface have open subsets which are smooth; this is indicated in the second half of the section. Finally, the same methods are used to show that bundles on a blowup which are topologically trivial on the exceptional divisor can be approximated (off a finite set) by bundles which are holomorphically trivial on the divisor.

In general, a semi-stable sheaf $\mathcal{S}$ on the compact surface $X$ determines a semi-stable bundle $\Sigma(\mathcal{S})$ admitting a Hermitian-Einstein connection as follows: $\Sigma(\mathcal{S}):=\Sigma\left(\mathcal{S}^{* *}\right)$ and if $\mathcal{A} \subset \mathcal{S}$ has $\mu(\mathcal{A})=\mu(\mathcal{S})$, then $\Sigma(\mathcal{S}):=$ $\Sigma(\mathcal{A}) \oplus \Sigma(\mathcal{S} / \mathcal{A})$. It is straightforward to verify by induction on rank that this prescription is well-defined and uniquely determines the bundle $\Sigma(\mathcal{S})$. This bundle has the same rank and determinant as $\mathcal{S}$ and never has greater charge; it is a direct sum of stable bundles all of the same slope (i.e., is quasistable), and there are non-zero holomorphic maps $\mathcal{S} \rightarrow \Sigma(\mathcal{S}), \Sigma(\mathcal{S}) \rightarrow \mathcal{S}^{* *}$.

It is also convenient to introduce the notation $B(E)$ for a semi-stable bundle $E$ to denote the set of points $x \in X$ for which there is a semi-stable bundle $A$ with $\mu(A)=\mu(E)$ and a sheaf inclusion $A \rightarrow E$ such that $A_{x} \rightarrow E_{x}$ not of maximal rank; (note that the quotient $E / A$ must be torsion-free else semi-stability of $E$ will be violated by the maximal normal extension of $A$ in $E$ ). Again, it is easily verified by induction on the rank of $E$ that $B(E)$ is finite.

Let $E$ be a semi-stable $r$-bundle on $X$, and let $A$ be a semi-stable $a$ bundle with $\mu(A)=\mu(E)$ for which there is a map $A \rightarrow E$ inducing a sheaf inclusion. Pick a point $x_{0} \in X \backslash B(E)$ and let $\widetilde{X} \stackrel{\pi}{\rightarrow} X$ be the blowup of $X$ at $x_{0}$. If $E_{1}$ is any $r$-bundle on a neighborhood of $L:=\pi^{-1}\left(x_{0}\right)$ and $\rho: E \rightarrow\left(\pi_{*} E_{1}\right)^{* *}$ is an isomorphism over this neighborhood, then if $E$ is in fact stable it follows from Proposition 3.4 that the bundle $\widetilde{E}:=E \#_{\rho} E_{1}$ is stable with respect to $\omega_{\epsilon}$ for all sufficiently small $\epsilon>0$; the more delicate and interesting case is when $E$ is not stable which is henceforth assumed.

Now take $E_{1}=\mathcal{O}(1) \oplus \mathcal{O}^{r-1}$. Up to isomorphism, the bundle $\widetilde{E}$ is determined by a non-zero element, $\varphi$ say, of the vector space $E_{x_{0}}^{*}$, with any non-zero multiple giving an isomorphic bundle: the correspondence is given explicitly by taking direct images of the sequence $0 \rightarrow \widetilde{E} \rightarrow \widetilde{E}(-1) \rightarrow$ $\left.\widetilde{E}(-1)\right|_{L} \rightarrow 0$ and using the fact that $\pi_{*} \widetilde{E}(-1)$ is locally free, hence equal to E. 
Let $\widetilde{A} \subset \widetilde{E}$ have torsion-free quotient $\widetilde{C}$ and satisfy $\mu\left(\pi_{*} \widetilde{A}\right)=\mu(E)$, and consider the commutative diagram

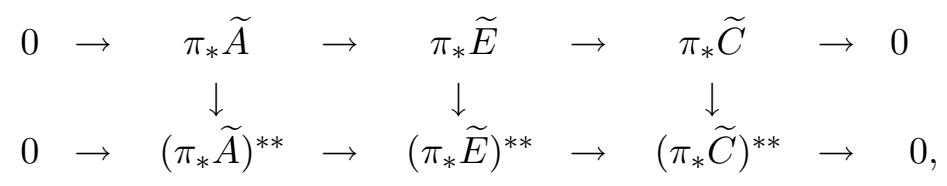

in which the vertical arrows are inclusions and the rows are not assumed to be exact. By taking direct images of the exact sequence $0 \rightarrow \widetilde{A} \rightarrow \widetilde{E} \rightarrow$ $\widetilde{C} \rightarrow 0$ and using the fact that $\pi_{*}^{1} \widetilde{E}=0$, it follows that the top row is exact except at $\pi_{*} \widetilde{C}$ where the cokernel of $\pi_{*} \widetilde{E} \rightarrow \pi_{*} \widetilde{C}$ is $\pi_{*}^{1} \widetilde{A}$. Since $x_{0} \notin B(E)$, $\left(\pi_{*} \widetilde{A}\right)^{* *} \subset\left(\pi_{*} \widetilde{E}\right)^{* *}=E$ is a sub-bundle at $x_{0}$ and the lower row must be exact near there, implying that $\left(\pi_{*} \widetilde{A}\right)^{* *} / \pi_{*} \widetilde{A} \rightarrow\left(\pi_{*} \widetilde{E}\right)^{* *} / \pi_{*} \widetilde{E}=\mathbb{C}$ is injective and that $\left(\pi_{*} \widetilde{E}\right)^{* *} / \pi_{*} \widetilde{E} \rightarrow\left(\pi_{*} \widetilde{C}\right)^{* *} / \pi_{*} \widetilde{C}$ is surjective.

Replace the top row of the diagram by $0 \rightarrow \pi_{*} \widetilde{A}(-1) \rightarrow \pi_{*} \widetilde{E}(-1) \rightarrow$ $\pi_{*} \widetilde{C}(-1) \rightarrow 0$, which is exact except possibly at $\pi_{*} \widetilde{C}(-1)$, with the cokernel of $\pi_{*} \widetilde{E}(-1) \rightarrow \pi_{*} \widetilde{C}(-1)$ being $\pi_{*} \widetilde{A}(-1)$. The lower row remains unchanged since $\left(\pi_{*} S(-1)\right)^{* *}=\left(\pi_{*} S\right)^{* *}$ for any torsion-free $S$.

Since $\widetilde{E}(-1)=\mathcal{O} \oplus \mathcal{O}(-1)^{r-1}$ near $L$, it follows $\pi_{*} \widetilde{E}(-1)$ is locally free. Since $\widetilde{C}$ is torsion-free, $\widetilde{A}$ is a subbundle of $\widetilde{E}$ at generic points of $L$ so if $\left.\widetilde{A}\right|_{L}=\oplus \mathcal{O}\left(a_{i}\right)$, each $a_{i}$ must be at most 1 ; therefore $\pi_{*} \widetilde{A}(-1)$ is locally free. Hence the first two vertical arrows are isomorphisms, implying that $\pi_{*} \widetilde{C}(-1)$ is locally free and $\pi_{*}^{1} \widetilde{A}(-1)=0$. By Lemma $2.2(\mathrm{f}), \widetilde{A}$ splits as a sum of the line bundles $\mathcal{O}$ and $\mathcal{O}(1)$ near $L$, and by the remark following the proof of Lemma $2.2(\mathrm{f}), \widetilde{C}$ is locally free and also splits in the same fashion. Since $\operatorname{det} \widetilde{E}=\mathcal{O}(1)$ near $L$, the only two possibilities are that near $L, \widetilde{A}$ is trivial and $\widetilde{C}=\mathcal{O}(1) \oplus \mathcal{O}^{c-1}$ or $\widetilde{A}=\mathcal{O}(1) \oplus \mathcal{O}^{a-1}$ and $\widetilde{C}$ is trivial. These two possibilities occur iff the kernel of $\varphi: E_{x_{0}} \rightarrow \mathbb{C}$ does or does not contain the image of $\left(\pi_{*} \widetilde{A}\right)_{x_{0}}^{* *}$ respectively. If it does not, then $\mu\left(\widetilde{A}, \omega_{\alpha}\right)=\mu\left(\pi_{*} \widetilde{A}\right)-\alpha / a=\mu(E)-\alpha / a<\mu(E)-\alpha / r=\mu\left(\widetilde{E}, \omega_{\alpha}\right)$, so $\widetilde{E}$ would be stable for all $\alpha>0$ sufficiently small if every such $\widetilde{A}$ could be guaranteed to fall into the second category.

In general, it is not be possible guarantee that $\left(\pi_{*} \widetilde{A}\right)_{x_{0}}^{* *}$ should not be contained in the kernel of $\varphi$. However, the following somewhat technical lemma shows that if the construction is repeated at a number of points, a uniform upper bound on the number of such "bad" points can be given:

Lemma 4.1. Suppose $x_{1}, \ldots, x_{n} \in X \backslash B(E)$. Then there are linear maps $\varphi_{i}: E_{x_{i}} \rightarrow \mathbb{C}, i=1, \ldots, n$ with the following property: For any stable bundle $A$ on $X$ of rank a with $\mu(A)=\mu(E)$ and any non-zero map $A \rightarrow E$, the number of maps $\varphi_{i}$ for which $A_{x_{i}} \subset \operatorname{ker} \varphi_{i}$ is at most $m-1$, where the multiplicity of $A$ in $\Sigma(E)$ is $m a-c, 0 \leq c<a$. 
Proof. By induction on the rank $r$ of $E$. Without loss of generality, there is a semi-stable bundle $K$ with $\mu(K)=\mu(E)$ and a map $K \rightarrow E$ such that the quotient $B$ is a torsion-free stable sheaf of rank $0<b<r$. By the inductive hypotheses, maps $\varphi_{i}^{K}$ with the requisite properties exist for $K$.

Suppose first that the extension $0 \rightarrow K \rightarrow E \rightarrow B \rightarrow 0$ is non-trivial. If $A$ is a stable bundle of rank $a$ with $\mu(A)=\mu(E)$ and there is a nonzero map $A \rightarrow E$, then by stability of $A$ and $B$ the composition $A \rightarrow B$ is either 0 or an isomorphism, but the latter is ruled out by the assumption that the extension does not split. Thus any such $A$ maps into $K$ and since $\Sigma(E)=\Sigma(K) \oplus B^{* *}$, any extensions of the maps $\varphi_{i}^{K}$ to $E_{x_{i}}$ will satisfy the requirements of the lemma.

Suppose now that the extension does split, so $B$ is in fact locally free. Fix maps $\varphi_{i}^{K}$ as above and choose maps $\varphi_{i}^{B}: B_{x_{i}} \rightarrow \mathbb{C}$; set $\varphi_{i}:=\varphi_{i}^{K}+\varphi_{i}^{B}$. If $\operatorname{Hom}(B, K)=0$ then for any $A$ as in the last paragraph, the map $A \rightarrow B$ is either 0 or an isomorphism and $A \rightarrow K$ is then an inclusion or 0 respectively, so the conclusion of the lemma is again satisfied. If, on the other hand, there does exist a non-zero homomorphism from $B$ into $K$, let $m b-c$ be the multiplicity of $B$ in $\Sigma(K)$ for some integers $m, c$ with $0 \leq c<b$. By the inductive hypothesis, for any non-zero map $B \rightarrow K$ the image of $B$ is contained in the kernels of at most $m-1$ of the maps $\varphi_{i}^{K}$.

Suppose then that for each choice of maps $\varphi_{i}^{B}: B_{x_{i}} \rightarrow \mathbb{C}$ there is a map $(\Psi, \psi): B \rightarrow K \oplus B$ such that more than $m-1$ of the maps $\varphi_{i}=\varphi_{i}^{K}+\varphi_{i}^{B}$ restricted to the image of $B$ in $K \oplus B$ are identically 0 . Since $B$ is stable, the map $\psi$ is a multiple of the identity, but this multiple cannot be 0 otherwise more than $m-1$ of the maps $\varphi_{i}^{K}$ restricted to the image of $B$ are 0 . After rescaling $(\Psi, \psi)$ it can be supposed that $\psi$ is the identity; then $\Psi$ must be uniquely determined by $\varphi_{1}^{B}, \ldots, \varphi_{n}^{B}$ since the non-zero difference between any two would give a map $B \rightarrow K$ whose images in $K_{x_{i}}$ would be contained in more than $m-1$ of the subspaces $\operatorname{ker} \varphi_{i}^{K}$. Hence $\Psi=\Psi\left(\varphi^{B}\right)$ where $\varphi^{B}:=\left(\varphi_{1}^{B}, \ldots, \varphi_{n}^{B}\right)$.

For each $\varphi^{B} \in \mathcal{B}:=B_{x_{1}}^{*} \backslash\{0\} \times \cdots \times B_{x_{n}}^{*} \backslash\{0\}$ the map $\Psi$ satisfies

$$
\varphi_{i}^{K} \cdot \Psi\left(\varphi^{B}\right)\left(x_{i}\right)=-\varphi_{i}^{B}, \quad i=i_{1}, \ldots, i_{m+k}
$$

where $k \geq 0$ depends on $\varphi^{B}$. If $\left\{\varphi_{\alpha}^{B}\right\}_{\alpha=1}^{\infty} \subset \mathcal{B}$ converges to $\varphi^{B} \in \mathcal{B}$, the sequence $\left\{\Psi\left(\varphi_{\alpha}^{B}\right)\right\}_{\alpha=1}^{\infty} \subset H^{0}(X, \operatorname{Hom}(B, K))$ must be bounded, otherwise $\left\{\Psi\left(\varphi_{\alpha}^{B}\right) /\left\|\Psi\left(\varphi_{\alpha}^{B}\right)\right\|\right\}$ has a subsequence converging to $\widetilde{\Psi} \in H^{0}(X, \operatorname{Hom}(B, K))$ with $\|\widetilde{\Psi}\|=1$ but with $\varphi_{i}^{K} \widetilde{\Psi}=0$ for more than $m-1$ values of $i$, a contradiction. If $\widehat{\Psi} \in H^{0}(X, \operatorname{Hom}(B, K))$ is the limit of any convergent subsequence of $\left\{\Psi\left(\varphi_{\alpha}^{B}\right)\right\}$ then for $i=i_{1}, \ldots, i_{m+k}$ (possibly depending on the subsequence) it follows $\varphi_{i}^{K} \cdot \widehat{\Psi}=-\varphi_{i}^{B}$, and by uniqueness, that $\widehat{\Psi}=\Psi\left(\varphi^{B}\right)$; hence $\Psi\left(\varphi^{B}\right)$ depends continuously on $\varphi^{B} \in \mathcal{B}$. 
For each subset of $\left\{x_{1}, \ldots, x_{n}\right\}$ consisting of at least $m$ points there is a corresponding subset of $\mathcal{B}$ consisting of those $\varphi^{B}$ for which (4.2) holds. Let $\mathcal{B}_{0}$ denote the union of those non-empty subsets for which (4.2) holds at the minimum number of points, $m+k_{0}$ say. If $\left\{\varphi_{\alpha}^{B}\right\}_{\alpha=1}^{\infty} \subset \mathcal{B} \backslash \mathcal{B}_{0}$ converges to $\varphi^{B} \in \mathcal{B}$, then each $\varphi_{\alpha}^{B}$ in some subsequence will satisfy (4.2) at the same set of points $\left\{x_{i_{1}}, \ldots, x_{i_{m+k}}\right\}$ and therefore $\Psi\left(\varphi^{B}\right)$ also satisfies the same set of equations, implying that $\varphi^{B} \notin \mathcal{B}_{0}$; hence $\mathcal{B}_{0}$ is open. Thus for some $i_{1}, \ldots, i_{m+k_{0}},(4.2)$ holds for all $\varphi^{B}$ in a non-empty open subset of $\mathcal{B}$, which easily implies that $\Psi\left(\varphi^{B}\right)$ is a linear function of $\varphi_{i_{1}}^{B}, \ldots, \varphi_{i_{m+k_{0}}}^{B}$. Moreover, it must be the case that $k_{0}=0$, else by taking $\varphi_{i}^{B}=0$ for $i \neq i_{1}$ with $\varphi_{i_{1}}^{B} \neq 0, \Psi\left(\varphi^{B}\right)$ is a non-zero map $B \rightarrow K$ whose image is contained in more than $m-1$ of the subspaces $\operatorname{ker}\left(\varphi_{i}^{K}\right)$.

Renumbering the points $x_{1}, \ldots, x_{n}$, it can be assumed that $\varphi^{K} \cdot \Psi\left(\varphi^{B}\right)=$ $-\varphi^{B}$ for $\varphi^{K}:=\left(\varphi_{1}^{K}, \ldots, \varphi_{m}^{K}\right)$ and $\varphi^{B}$ is now equal to $\left(\varphi_{1}^{B}, \ldots, \varphi_{m}^{B}\right)$, where it is understood that $\Psi$ is evaluated at each point $x_{i}$ for each term respectively in this matrix equation.

The map $\Psi$ can be viewed as a homomorphism $B \otimes \oplus_{i=1}^{m} B_{x_{i}}^{*} \rightarrow K$. Since $B$ is stable and $K$ is semi-stable of the same slope as $B$, the kernel of this homomorphism must be of the the form $B \otimes V$ for some vector subspace $V \subset \oplus_{i=1}^{m} B_{x_{i}}^{*}$, but the equation $\varphi^{K} \cdot \Psi\left(\varphi^{B}\right)=-\varphi^{B}$ implies that $V$ must have dimension 0 . Thus $\Psi: B \otimes \oplus_{i=1}^{m} B_{x_{i}}^{*} \rightarrow K$ is a sheaf inclusion, and indeed since $B(K) \subset B(E)$, this map must have maximal rank at each point $x_{i}, i=1, \ldots, m$. It follows that the multiplicity of $B$ in $K$ is at least $m b$, and since this multiplicity is by assumption $m b-c$, it follows that $c=0$. Thus the multiplicity of $B$ in $E=K \oplus B$ is $m b+1=(m+1) b-(b-1)$, and the preceding argument shows that for generic $\varphi_{i}^{B}$, the homomorphisms $\varphi_{i}$ have the desired property.

Suppose that $E$ is as above and that $\Sigma(E)=\bigoplus_{i} V_{i} \otimes A_{i}$ where $V_{i}$ is a $d_{i}$-dimensional vector space and $A_{i}$ is a stable $a_{i}$-bundle with $\mu\left(A_{i}\right)=\mu(E)$ and with $A_{i} \not 千 A_{j}$ for $i \neq j$. Pick any $n$ points $x_{1}, \ldots, x_{n} \in X \backslash B(E)$ and glue in the bundle $E_{1}$ at each of these points in the generic fashion described by Lemma 4.1 to obtain a bundle $\widetilde{E}$ on the blowup $\widetilde{X} \stackrel{\pi}{\rightarrow} X$ of $X$ at all $x_{1}, \ldots, x_{n}$. If $\sigma_{i}$ represents $-L_{i}$ let $\rho:=\sum_{i} \sigma_{i}$ and for $\epsilon>0$ take $\omega_{\epsilon}:=\pi^{*} \omega+\epsilon \rho$.

Let $\widetilde{A} \subset \widetilde{E}$ have rank $a$, have torsion-free quotient, and maximize $\mu\left(*, \omega_{\epsilon}\right)$ over the admissible subsheaves of $\widetilde{E}$ by $\widetilde{A}$ for all $\epsilon>0$ sufficiently small. Since $\mu\left(\widetilde{A}, \omega_{\epsilon}\right)=\mu\left(\pi_{*} \widetilde{A}\right)+\epsilon \rho \cdot c_{1}(\widetilde{A}) / a$, letting $\epsilon \rightarrow 0$ shows that $\mu\left(\pi_{*} \widetilde{A}\right)=$ $\mu(E)$. By the discussion preceding Lemma $4.1, \sigma_{i} \cdot c_{1}(\widetilde{A})$ is either 0 or -1 according respectively to whether or not $\left(\pi_{*} \widetilde{A}\right)^{* *}$ is contained in the kernel of the linear form defining the gluing of $E$ to $E_{1}$ at $x_{i}$. By Lemma 4.1 itself, the former occurs at $\operatorname{most}=:=\max _{i}\left\{d_{i} / a_{i}\right\}(\leq r)$ times. Since 


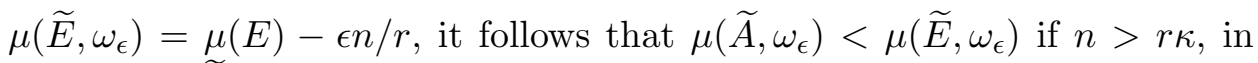
which case $\widetilde{E}$ is stable with respect to $\omega_{\epsilon}$ for all $\epsilon>0$ sufficiently small.

To summarise:

Proposition 4.3. Let $E$ be a semi-stable $r$-bundle with $\Sigma(E)=\bigoplus_{i} V_{i} \otimes A_{i}$ where $V_{i}$ is a $d_{i}$-dimensional vector space and $A_{i}$ is a stable $a_{i}$-bundle with $\mu\left(A_{i}\right)=\mu(E)$ and with $A_{i} \not A_{j}$ for $i \neq j$. If $n>r\left[\max _{i}\left\{d_{i} / a_{i}\right\}\right]$ then for any choice of $n$ points in $X \backslash B(E)$ there is a bundle $\widetilde{E}$ on the blowup $\widetilde{X} \stackrel{\pi}{\rightarrow} X$ of $X$ at these points such that $\widetilde{E}$ restricts to $\mathcal{O}(1) \oplus \sum_{1}^{r-1} \mathcal{O}$ on each component of the exceptional divisor, $\left(\pi_{*} \widetilde{E}\right)^{* *}=E$, and $\widetilde{E}$ is stable with respect to $\omega_{\epsilon}:=\pi^{*} \omega+\epsilon \sum_{i=1}^{n} \sigma_{i}$ for all $\epsilon>0$ sufficiently small.

\section{Remarks.}

1. Since $\pi_{*} \widetilde{E}(-1)$ is locally free, there is a map $\pi^{*} E \rightarrow \widetilde{E}(-1)$ obtained by pulling-back and composing with the canonical map $\pi^{*} \pi_{*} \widetilde{E}(-1) \rightarrow \widetilde{E}(-1)$. Similarly, since $\pi_{*}\left(\widetilde{E}^{*}\right)$ is locally free, (after dualising) there is a map $\widetilde{E} \rightarrow$ $\pi^{*} E$, and the composition $\pi^{*} E(1) \rightarrow \widetilde{E} \rightarrow \pi^{*} E$ is $s \mathbf{1}_{E}$ where $s \in \Gamma(\mathcal{O}(-1))$ defines the exceptional divisor.

2. If $r=2$ the entire procedure is considerably simplified. In particular, if $E_{0}$ is given by an extension $0 \rightarrow L_{1} \rightarrow E_{0} \rightarrow L_{2} \otimes \mathcal{J} \rightarrow 0$ for some line bundles $L_{1}, L_{2}$ with $\operatorname{deg}\left(L_{1}\right)=\operatorname{deg}\left(L_{2}\right)$ and some sheaf of ideals $\mathcal{J}$ with $\mathcal{O} / \mathcal{J}$ supported at a finite set, then if $L_{1} \not L_{2}$ or the extension does not split, it suffices to blow up at one point and take $\widetilde{E}$ to be a non-trivial extension $0 \rightarrow \pi^{*} L_{1}(1) \rightarrow \widetilde{E} \rightarrow \pi^{*} L_{2} \otimes \mathcal{J} \rightarrow 0$. If $E_{0}=L_{1} \oplus L_{1}$ then it suffices to blow up at 3 points and take a non-zero extension $0 \rightarrow \pi^{*} L_{1}(1,-1,1) \rightarrow$ $\widetilde{E} \rightarrow \pi^{*} L_{1}(0,0,0) \rightarrow 0$.

3. Instead of gluing-in the bundle $E_{1}=\mathcal{O}(1) \oplus \mathcal{O}^{r-1}$ to construct $\widetilde{E}$, another natural choice is to glue in the bundle $\mathcal{O}(1) \oplus \mathcal{O}(-1) \oplus \mathcal{O}^{r-2}$ (assuming of course that $r \geq 2$ ). The new bundle now has the same first Chern class (and determinant) as $E_{0}$, and charge one unit greater (as opposed to $(r-1) / 2 r$ units greater). An analysis similar to that which was given above to prove Proposition 4.3 should be possible in this case, but the calculations are more involved and as yet have not been carried out.

As mentioned in the introduction to this section, the gluing construction also yields a simple way to by-pass the technical difficulties of [D5] which can occur because of singularities in moduli spaces of stable bundles.

For a stable $r$-bundle $E$ on the compact surface $(X, \omega)$ with HermitianEinstein connection $\nabla$, the cokernel of $\nabla_{+}: \Lambda^{1}\left(\operatorname{End}_{0} E\right) \rightarrow \Lambda_{+}^{2}\left(\operatorname{End}_{0} E\right)$ vanishes iff $H^{2}\left(X\right.$, End $\left._{0} E\right)$ does, where End ${ }_{0}$ denotes trace-free endomorphisms. Thus $\nabla$ is a smooth point in the moduli space of irreducible Hermitian-Einstein connections iff $E$ is a smooth point in the moduli space of 
stable bundles. By Serre duality, $H^{2}\left(X, \operatorname{End}_{0} E\right) \simeq H^{0}\left(X, \text { End }_{0} E \otimes K_{X}\right)^{*}$ where $K_{X}$ is the canonical bundle of $X$. If $s \in H^{0}\left(X\right.$, End $\left.{ }_{0} E \otimes K_{X}\right)$ is a non-zero section, pick a point $x_{0}$ at which $s$ is not zero, and blow up $X$ at $x_{0}$. Now take $E_{0}=E, E_{1}=\mathcal{O}(-1) \oplus \mathcal{O}(1) \oplus \mathcal{O}^{r-2}$ and construct a bundle $\widetilde{E}=E_{0} \#_{\rho} E_{1}$ on $\widetilde{X}$ as in $\S 2$. Since $K_{\widetilde{X}} \simeq\left(\pi^{*} K_{X}\right)(-1)([\mathbf{B P V}$, Theorem I.9.1]), Lemma $2.2\left(\right.$ a) implies that $\pi_{*}\left(\operatorname{End}_{0} \widetilde{E} \otimes K_{\widetilde{X}}(-1)\right)$ is locally free, and therefore it is isomorphic to End ${ }_{0} E \otimes K_{X}$ since it agrees with this sheaf away from $x_{0}$. The direct image of the sequence

$0 \rightarrow \operatorname{End}_{0} \widetilde{E} \otimes K_{\widetilde{X}} \rightarrow \operatorname{End}_{0} \widetilde{E} \otimes K_{\widetilde{X}}(-1) \rightarrow \operatorname{End}_{0} \widetilde{E} \otimes K_{\widetilde{X}} \otimes \mathcal{O}_{L_{0}}(-1) \rightarrow 0$ thus gives $0 \rightarrow \pi_{*}$ End $_{0} \widetilde{E} \otimes K_{\widetilde{X}} \rightarrow \operatorname{End}_{0} E \otimes K_{X} \rightarrow \mathbb{C}_{x_{0}}$, and it is straightforward to check that the under the composition $H^{0}\left(X\right.$, End $\left.{ }_{0} E \otimes K_{X}\right) \simeq$ $H^{0}\left(\widetilde{X}, \operatorname{End}_{0} \widetilde{E} \otimes K_{\widetilde{X}}(-1)\right) \rightarrow H^{0}\left(L_{0}, \widetilde{E} \otimes K_{\widetilde{X}}(-1)\right) \simeq \mathbb{C}$ the section $s$ is not mapped to zero for generic $\rho$. For such $\rho$, it follows that the dimension of $H^{2}\left(\widetilde{X}, \operatorname{End}_{0} \widetilde{E}\right)$ is one less than that of $H^{2}\left(X, \operatorname{End}_{0} E\right)$ so after performing this operation at enough points the bundle $\widetilde{E}$ on $\widetilde{X}$ will satisfy $H^{2}\left(\widetilde{X}\right.$, End $\left._{0} \widetilde{E}\right)=0$. By semi-continuity of cohomology the same will be true for any bundle on $\widetilde{X}$ sufficiently near $\widetilde{E}$, and by Proposition 3.4 the bundle $\widetilde{E}$ will be stable with respect to $\omega_{\alpha}$ for all suitable $\alpha$ sufficiently close to 0 .

More generally, if $L$ is any line bundle on $X$ the same technique shows that by blowing up at least $h^{2}\left(\left(\right.\right.$ End $\left.\left._{0} E\right) \otimes L\right)$ generic points in $X$, the generic bundle $\widetilde{E}=E \#_{\rho} E_{1}$ on the blowup satisfies $H^{2}\left(\left(\operatorname{End}_{0} \widetilde{E}\right) \otimes \pi^{*} L\right)=0$.

To summarize:

Proposition 4.4. If $E$ is an $r$-bundle on $X$ and $L$ is a holomorphic line bundle, then for any set $T$ of $n \geq h^{2}\left(X,\left(\operatorname{End}_{0} E\right) \otimes L\right)$ points in general position there is a blowup $\widetilde{X}$ of $X$ centered at $T$ together with a bundle $\widetilde{E}$ on $\widetilde{X}$ restricting to $\mathcal{O}(1) \oplus \mathcal{O}(-1) \oplus \mathcal{O}^{r-2}$ on each component of the exceptional divisor satisfying $\left(\pi_{*} \widetilde{E}\right)^{* *}=E$ and $H^{2}\left(\widetilde{X},\left(\operatorname{End}_{0} \widetilde{E}\right) \otimes \pi^{*} L\right)=0$.

If $X^{\prime}$ is a blowup of $X$ with exceptional divisor $D^{\prime}$, and $E^{\prime}$ is a bundle on $X^{\prime}$ which is topologically trivial on $D^{\prime}$, the cokernel of $H^{1}\left(X^{\prime}\right.$, End $\left.E_{0} E^{\prime}\right) \rightarrow$ $H^{1}\left(D^{\prime}\right.$, End $\left._{0} E^{\prime}\right)$ is the kernel of the epimorphism $H^{2}\left(X^{\prime},\left(\operatorname{End}_{0} E^{\prime}\right)\left(-D^{\prime}\right)\right)$ $\rightarrow H^{2}\left(X^{\prime}\right.$, End $\left._{0} E^{\prime}\right)$. If the former group vanishes, every small deformation of $E^{\prime}$ on $D^{\prime}$ is induced by a small deformation of $E^{\prime}$ on $X^{\prime}$. Since every bundle on $\mathbb{P}_{1}$ with 0 first Chern class has arbitrarily small deformations which are holomorphically trivial, it follows in this case that $E^{\prime}$ has arbitrarily small deformations which are holomorphic pull-backs from $X$.

If $\operatorname{deg}\left(K_{X}, \omega\right)<0$ and $E^{\prime}$ is $\omega_{\alpha}$-stable for sufficiently small suitable $\alpha$, End $_{0} E^{\prime} \otimes K_{X^{\prime}}\left(D^{\prime}\right)$ has negative degree with respect to $\omega_{\alpha}$ and therefore $H^{2}\left(X^{\prime},\left(\right.\right.$ End $\left.\left._{0} E^{\prime}\right)\left(-D^{\prime}\right)\right)=0$ by duality. If $\operatorname{deg}\left(K_{X}, \omega\right) \geq 0$, applying 
Proposition 4.4 (blowing up at points of $X^{\prime} \backslash D^{\prime}$ ) yields a blowup $\widetilde{X}^{\prime}$ of $X^{\prime}$ with exceptional divisor $D$ together with a bundle $\widetilde{E}^{\prime}$ on $\widetilde{X}^{\prime}$ such that $H^{2}\left(\widetilde{X}^{\prime},\left(\right.\right.$ End $\left.\left._{0} \widetilde{E}^{\prime}\right)\left(-D^{\prime}\right)\right)=0$. From the previous paragraph it follows that there are arbitrarily small deformations of $\widetilde{E}^{\prime}$ which restrict to holomorphically trivial bundles on $D^{\prime}$. The behavior of the deformations on $D$ cannot be controlled, but for sufficiently small perturbations of $\widetilde{E}^{\prime}$, the bundles must be either trivial or split as $\mathcal{O}(1) \oplus \mathcal{O}(-1) \oplus \mathcal{O}^{r-2}$ on each component, by semi-continuity of cohomology.

\section{Structure of moduli spaces II.}

Let $X$ be a compact complex surface, equipped with a $\bar{\partial} \partial$-closed positive $(1,1)$-form $\omega$. Consider a sequence $\left\{E_{i}\right\}$ of stable holomorphic bundles of fixed topological type and degree, identified with a sequence $\left\{A_{i}\right\}$ of Hermitian-Einstein connections on a fixed unitary bundle. For this sequence of connections, the $L^{2}$ norms of the curvatures are uniformly bounded, so by Uhlenbeck's theorem [Sed], [U1] there is a finite set $S \subset X$ and gauge transformations such that the gauge-transformed sequence (also denoted $\left\{A_{i}\right\}$ ) converges weakly in $L_{1 \text {,loc }}^{2}(X \backslash S)$ to a connection $A$ defining a finite-action Hermitian-Einstein connection. Ellipticity of the Hermitian-Einstein equations combined with Donaldson's argument in the proof of Corollary 23 of [D2] shows that a subsequence is converging weakly in $L_{1, \text { loc }}^{p}(X \backslash S)$ for any $p$ and by bootstrapping and diagonalisation, that a subsequence converges strongly in $C^{k}$ on compact subsets of $X \backslash S$ for any $k$. Since a HermitianEinstein connection can be twisted locally by a Hermitian-Einstein connection on a trivial bundle so that the new connection has $\lambda=0$, it follows from the Removability of Singularities theorem [U2] that the limit extends across $S$ to define a new Hermitian-Einstein connection on a bundle over $X$, and therefore a new semi-stable bundle $E$ with $\Sigma(E)=E$. The new holomorphic bundle $E$ has the same rank and first Chern class as the bundles in the sequence, its determinant is the limit of the determinants, but its charge is at least one less for each point in $S$ where the curvature has "bubbled".

Following [D5], this type of "convergence" for sequences of connections is referred to as weak convergence (on $X \backslash S$ ), and sequences of stable holomorphic bundles of the same degree and topological type converge weakly (on $X \backslash S$ ) (with respect to $\omega$ ) if the corresponding irreducible HermitianEinstein connections converge weakly.

In dealing with limits of sequences of stable bundles, arguments are greatly simplified whenever it is known that a weak limit is itself stable, rather than just semi-stable. The stabilization construction given in the previous section is designed to meet this type of need, and when combined with Lemma 2.2 of [B5] (semi-continuity of cohomology on $H^{0}$ for weak limits), the upshot is Lemma 5.1 below. Recall that the notation $\Lambda F$ denotes $*(\omega \wedge F)$ and if 
$A$ is a connection on a unitary bundle and $g$ is a complex automorphism (an intertwining operator) on that bundle, $g \cdot A$ is the connection with $(0,1)$ part $\bar{\partial}_{g \cdot A}=g \circ \bar{\partial}_{A} \circ g^{-1}$ and $(1,0)$ part $\partial_{g \cdot A}=\left(g^{*}\right)^{-1} \circ \partial_{A} \circ g^{*}$.

Lemma 5.1. Let $\left\{E_{i}\right\}$ be a sequence of stable bundles on $X$ corresponding to a sequence $\left\{A_{i}\right\}$ of Hermitian-Einstein connections on a fixed $U(r)$-bundle and let $S \subset X$ be a finite set such that the sequence converges weakly on $X \backslash S$ to a Hermitian-Einstein connection A defining a quasi-stable bundle E. If $(\widetilde{X}, \widetilde{E})$ is a stabilization of $E$ with the none of the blown-up points lying in $S$, then there is a subsequence with stabilizations $\widetilde{E}_{i_{j}}$ on $\widetilde{X}$ converging weakly to $\widetilde{E}$ on $\widetilde{X} \backslash \pi^{-1}(S)$. Here stability on $\widetilde{X}$ is with respect to $\omega_{\epsilon}=\omega_{\epsilon \alpha_{0}}$ for $\alpha_{0}:=(1, \ldots, 1)$ and any $\epsilon>0$ sufficiently small.

Proof. Let $T \subset X \backslash B(E)$ be the finite set used to stabilize $E$. Since there is no bubbling of curvature near the points of $T$, a sequence of integrable connections $\widetilde{A}_{i}$ corresponding to bundles $\widetilde{E}_{i}$ with $\left(\pi_{*} \widetilde{E}_{i}\right)^{* *}=E_{i}$ can be found such that $\widetilde{A}_{i}$ agrees with $\pi^{*} A_{i}$ outside a fixed neighborhood $\widetilde{U}$ of the exceptional divisor and which converge smoothly inside this neighborhood to a connection inducing $\widetilde{E}$ there, so the $\widetilde{A}_{i}$ converge weakly in $L_{1, \text { loc }}^{p}\left(\widetilde{X} \backslash \pi^{-1}(S)\right)$ to a connection inducing $\widetilde{E}$.

If $b_{1}(X)$ is even then by Proposition 3.5, all of the bundles $\widetilde{E}_{i}$ and $\widetilde{E}$ will be stable with respect to the same metric $\omega_{\epsilon}$ for sufficiently small $\epsilon>0$. However, by construction of $\widetilde{E}$ and $\widetilde{E}_{i}$, a reflexive subsheaf $\widetilde{A}_{i}$ minimising $\nu_{\widetilde{E}_{i}}\left(*, \omega_{\epsilon}\right)$ for all sufficiently small $\epsilon$ and sufficiently large $i$ will satisfy the same type of splitting behavior as that described in the discussion preceding Proposition 4.3 , and therefore there exists $\epsilon_{0}>0$ such that $\widetilde{E}_{i}$ is $\omega_{\epsilon}$-stable for all $i$ and all $\epsilon<\epsilon_{0}$, regardless of the parity of $b_{1}(X)$.

By weak compactness on $\widetilde{X}$ there is a finite set $\widetilde{S} \subset \widetilde{X}$ such that, after gauge transformations a subsequence of the corresponding $\omega_{\epsilon}$-HermitianEinstein connections $\widetilde{A}_{i}^{\prime}$ inducing $\widetilde{E}_{i}$ converges weakly in $L_{1, \text { loc }}^{p}(\widetilde{X} \backslash \widetilde{S})$ to a Hermitian-Einstein $\widetilde{A}^{\prime}$ connection on $\widetilde{X}$ which, after removal of singularities, defines a semi-stable bundle $\widetilde{E}^{\prime}$ there. By Lemma 2.2 of [B5], after rescaling if necessary, the automorphisms $g_{i}$ intertwining $\widetilde{A}_{i}^{\prime}$ with $\widetilde{A}_{i}$ give rise to a nonzero holomorphic map $\widetilde{E} \rightarrow \widetilde{E}^{\prime}$, but since the former is stable and the two bundles have the same degree, it must be an isomorphism. This implies that the sequences $\left\{g_{i}\right\},\left\{g_{i}^{-1}\right\}$ are both uniformly bounded in $C^{0}(\widetilde{X})$, and by Lemma 2.1 of that same reference it follows that $\widetilde{S}=\pi^{-1}(S)$ and that the two sequences bubble the same amount of charge at each point of $S$.

From the complex analytic viewpoint, Propositions 3.4 and 3.5 indicate that on a blowup $\widetilde{X}$ of $X$ there exist "stable" moduli spaces $\mathcal{M}\left(\widetilde{X}, E_{\text {top }}, \omega_{\epsilon \alpha}\right)$ of stable bundles as $\epsilon$ tends to 0 , at least when $b_{1}(X)$ is even. Given a 
bundle $\widetilde{E}$ in one of these moduli spaces, it is natural to enquire about the behavior of the corresponding sequences of Hermitian-Einstein connections and to determine the extent to which the behavior of this sequence reflects the complex analytic picture of a bundle on $X$ glued to a bundle on a neighborhood of the exceptional divisor according the prescription of $\S 2$. These questions are at least partially answered by Corollary 5.3 below where it is shown that the corresponding connections converge off the blown-up set and $B\left(\pi_{*} E\right)$ to the Hermitian-Einstein connection on $\Sigma\left(\pi_{*} E\right)$; the behavior of the sequence near the exceptional divisor is described in Theorem 5.4.

Proposition 5.2. Let $\widetilde{X} \stackrel{\pi}{\rightarrow} X$ be a modification of $X$ with exceptional divisor $D \subset \widetilde{X}$, and let $\left\{\omega_{i}\right\}$ be a sequence of $\bar{\partial} \partial$-closed positive $(1,1)$-forms on $\widetilde{X}$ converging smoothly to $\pi^{*} \omega$. Let $\widetilde{E}$ be a holomorphic r-bundle on $\widetilde{X}$ and let $\left\{A_{i}\right\}$ be a sequence of smooth hermitian connections on $\widetilde{E}$ such that

(i) $\left\|\Lambda_{i} F\left(A_{i}\right)-\sqrt{-1} \lambda_{i} \mathbf{1}\right\|_{L^{1}\left(\widetilde{X}, \omega_{i}\right)} \rightarrow 0$, for $\lambda_{i}=-2 \pi \mu\left(\widetilde{E}, \omega_{i}\right) / \operatorname{Vol}\left(\widetilde{X}, \omega_{i}\right)$, and

(ii) there is a finite set $S \subset X, p>4$ and $k \geq 1$ such that $\pi_{*} A_{i}$ converges weakly in $L_{k, \text { loc }}^{p}(X \backslash(S \cup \pi(D)), \omega)$ to a connection $A_{\infty}$ with $\left\|F\left(A_{\infty}\right)\right\|_{L^{2}(X)}<\infty$.

\section{Then}

(a) $\pi_{*} \widetilde{E}$ is semi-stable;

(b) the quasi-stable bundle $E_{\infty}$ on $X$ defined by $A_{\infty}$ (after removal of singularities) is isomorphic to $\Sigma\left(\pi_{*} \widetilde{E}\right)$;

(c) after suitable gauge transformations, a subsequence of $\left\{\pi_{*} A_{i}\right\}$ converges weakly in $L_{k}^{p}$ and strongly in $C^{k-1}$ on compact subsets of $X \backslash\left(\pi(D) \cup B\left(\left(\pi_{*} \widetilde{E}\right)^{* *}\right)\right)$.

Proof. If $L$ is an irreducible component of $D$, the curvature form $f_{i}$ of the Hermitian-Einstein connection on $\mathcal{O}(L)$ corresponding to the metric $\omega_{i}$ satisfies $\left\|f_{i}\right\|_{L^{2}\left(\widetilde{X}, \omega_{i}\right)}^{2}=4 \pi^{2}\left[1+\operatorname{deg}\left(\mathcal{O}(L), \omega_{i}\right)^{2} / V_{i}\right]$ for $V_{i}:=\operatorname{Vol}\left(\widetilde{X}, \omega_{i}\right)$, which converges to $4 \pi^{2}$. Ellipticity of the Hermitian-Einstein equations and the convergence of the sequence $\left\{\omega_{i}\right\}$ to $\pi^{*} \omega$ implies that (a subsequence of) $\left\{f_{i}\right\}$ converges weakly in $L^{2}(X)$ and smoothly on compact subsets of $X \backslash \pi(L)$ to a finite action solution of the Hermitian-Einstein equations which, by removable singularities, extends to $X$ to define a holomorphic line bundle of degree 0 there. By Lemma 2.2 of [B5] and Hartogs' Theorem, this line bundle has a non-zero holomorphic section, but since the degree of the bundle is 0 , this section is covariantly constant and therefore defines a global trivialisation - all of the curvature has concentrated along $L$. It follows from this that if $\widetilde{E}$ is twisted by a suitable line bundle trivial off $D$ and the sequence $\left\{A_{i}\right\}$ is correspondingly twisted by the sequence of Hermitian-Einstein connections 
on these line bundles, the hypotheses of the proposition remain true for the new sequence and it can be supposed from now on that $\pi_{*} \widetilde{E}$ is locally free.

By Lemma 2.2 of [B5] and Hartogs' Theorem again there is a non-zero holomorphic map $E_{\infty} \rightarrow \pi_{*} \widetilde{E}$, so if either bundle is stable this map is an isomorphism. In particular, this occurs if $\widetilde{E}$ is a line bundle.

(a) If $A \subset \pi_{*} \widetilde{E}$ has rank $a$ and torsion-free quotient then $\nu_{\pi_{*} \widetilde{E}}(A)=$ $\nu_{\widetilde{E}}\left(\pi^{*} A, \omega_{i}\right)-\frac{1}{r}\left(\omega_{i}-\pi^{*} \omega\right) \cdot \operatorname{det}\left(\widetilde{E} \otimes \pi^{*} A^{*}\right)$. Since $\nu_{\widetilde{E}}\left(\pi^{*} A, \omega_{i}\right) \geq \nu_{\widetilde{E}}\left(\widehat{A}, \omega_{i}\right)$ where $\widehat{A}$ is the maximal normal extension of $\pi^{*} A$ in $\widetilde{E}$ and the latter term has non-negative limit by hypothesis (i) and (1.7), the convergence of $\omega_{i}$ to $\pi^{*} \omega$ implies $\nu_{\pi_{*} \widetilde{E}}(A) \geq 0$.

(b) If either $E_{\infty}$ or $\pi_{*} \widetilde{E}$ is stable, this is proved already by the remarks above. If $\pi_{*} \widetilde{E}$ is not stable, there exists a subsheaf $A \subset \pi_{*} \widetilde{E}$ with torsion free quotient $C$ such that $\nu_{\pi_{*} \widetilde{E}}(A)=0$. Pulling back to $\widetilde{X}$ and taking the maximal normal extension gives a subsheaf $\widehat{A} \subset \widetilde{E}$ with torsion-free quotient $\widehat{C}$ such that $\pi_{*} \widehat{A}=A$ and $C \subset \pi_{*} \widehat{C}$. Desingularize the sequence $0 \rightarrow \widehat{A} \rightarrow \widetilde{E} \rightarrow \widehat{C} \rightarrow 0$ as in $\S 3$ of [B3] to obtain a modification $\widetilde{X}^{\prime} \stackrel{\pi^{\prime}}{\rightarrow} \widetilde{X} \stackrel{\pi}{\rightarrow} X$ and a sequence of bundles $0 \rightarrow \widehat{A}^{\prime} \rightarrow \pi^{*} \widetilde{E} \rightarrow \widehat{C}^{\prime} \rightarrow 0$ on $\widetilde{X}^{\prime}$ with $\pi_{*}^{\prime} \widehat{A}^{\prime}=\widehat{A}$ and $\widehat{C} \subset \pi_{*}^{\prime} \widehat{C}^{\prime}$.

Choose metrics $\omega_{i}^{\prime}=\pi^{\prime *} \omega_{i}+\rho_{\alpha_{i}}$ on $\widetilde{X}^{\prime}$ with $\rho_{\alpha_{i}}$ converging smoothly to 0 , and make this convergence sufficiently fast so that $\omega_{i} \wedge^{\prime} F\left(\pi^{\prime *} A_{i}\right)-$ $\sqrt{-1} \lambda_{i}^{\prime} \mathbf{1} \omega_{i}^{\prime 2}$ converges to 0 in $L^{1}\left(\widetilde{X}^{\prime}, \omega_{i}^{\prime}\right)$; this is possible since the connections $A_{i}$ are smooth (cf. the final remark in $\S 2$ of [B3, p. 631]). From (1.7) and (i) it follows that if $\beta_{i}$ is the second fundamental form of $\widehat{A}^{\prime}$ in $\pi^{* *} \widetilde{E}$ for the hermitian connection $\pi^{* *} A_{i}$, then $\beta_{i}$ is converging to 0 in $L^{2}\left(\widetilde{X}^{\prime}, \omega_{i}^{\prime}\right)$. This implies that the hypotheses of the proposition are satisfied by the induced connections on $\widehat{A}^{\prime}$ and $\widehat{C}^{\prime}$. If $A_{\infty}, C_{\infty}$ are the holomorphic bundles on $X$ defined by the limiting (Hermitian-Einstein) connections on $\widehat{A}^{\prime}, \widehat{C}^{\prime}$, then since $\beta_{i} \rightarrow 0$ it follows from the construction that $E_{\infty}=A_{\infty} \oplus C_{\infty}$. By induction on rank, $A_{\infty}=\Sigma\left(\left(\pi \pi^{\prime}\right)_{*} \widehat{A}^{\prime}\right)$ and $C_{\infty}=\Sigma\left(\left(\pi \pi^{\prime}\right)_{*} \widehat{C}^{\prime}\right)$, giving $E_{\infty}=A_{\infty} \oplus C_{\infty}=\Sigma\left(\left(\pi \pi^{\prime}\right)_{*} \widehat{A}^{\prime}\right) \oplus \Sigma\left(\left(\pi \pi^{\prime}\right)_{*} \widehat{C}^{\prime}\right)=\Sigma(A) \oplus \Sigma(C)=\Sigma\left(\pi_{*} \widetilde{E}\right)$, proving (b) in general.

(c) By passing to a subsequence it can be supposed that the sequence $\left\{g_{i}\right\}$ of intertwining operators from which the holomorphic map $\pi_{*} \widetilde{E} \rightarrow E_{\infty}$ is constructed is converging strongly in $C^{k}$ on any compact subset of $X \backslash(S \cup$ $\pi(D))$. If $\pi_{*} \widetilde{E}$ is stable then this map is an isomorphism and the sequence $\left\{g_{i}^{-1}\right\}$ must also be bounded in $C^{k}$ on compact subsets of $X \backslash(S \cup \pi(D))$. Then Lemma 2.1 of [B4] implies that curvature can only concentrate on $\pi(D)$ itself, which proves (c) in this case. The general case now follows by 
induction on rank using the proof of (b) when $\pi_{*} \widetilde{E}$ is not stable, since any point of $\widetilde{X}$ in the center of the modification $\widetilde{X}^{\prime} \rightarrow \widetilde{X}$ is mapped into $B\left(\pi_{*} \widetilde{E}\right)$ by $\pi$.

Corollary 5.3. If $\widetilde{E}$ on $\widetilde{X}$ is quasi-stable with respect to $\omega_{\alpha_{i}}$ for some suitable $\alpha_{i} \in \mathbb{R}_{+}^{n}$ converging to 0 , then for any fixed $k$, after suitable gauge transformations, a subsequence of the corresponding sequence $\left\{\nabla_{i}\right\}$ of HermitianEinstein connections converges uniformly in $C^{k}$ on compact subsets of $X \backslash\left(\pi(D) \cup B\left(\left(\pi_{*} \widetilde{E}\right)^{* *}\right)\right)$ to a Hermitian-Einstein connection inducing $\Sigma\left(\pi_{*} \widetilde{E}\right)$.

Proof. On any compact subset of $X \backslash \pi(D)$ the $L^{2}$ norm of $F\left(\nabla_{i}\right)$ with respect to $\omega$ is uniformly bounded. The weak compactness arguments of [Sed], [U1] still apply in this setting to obtain a subsequence of $\left\{\nabla_{i}\right\}$ (after gauge transformations) converging weakly in $L_{1}^{2}$ on compact subsets of $X \backslash(S \cup \pi(D))$ for some finite set $S \subset X$. Ellipticity of the Hermitian-Einstein equations together with boot-strapping and diagonalisation then give a subsequence converging in $C^{k}$ on compact subsets of this complement, and the conclusion then follows from the proposition.

Corollary 5.3 describes the behavior of sequences of Hermitian-Einstein connections away from the exceptional divisor $D$. To describe the behavior near $D$ consider the case that $\widetilde{X}$ is the blowup of $X$ at a single point $x_{0}$ and fix a metric on $\widetilde{X}$ of the form $\omega_{\epsilon}=\pi^{*} \omega+\epsilon \sigma$. If $\widetilde{E}$ is a holomorphic $r$ bundle on $\widetilde{X}$ which is $\omega_{\epsilon}$-stable for for every $\epsilon>0$ sufficiently small, then as $\epsilon \rightarrow 0$ the corresponding sequence $\left\{\nabla_{\epsilon}\right\}$ of Hermitian-Einstein connections converges on compact subsets of $X \backslash\left\{x_{0}\right\}$ to a Hermitian-Einstein connection on $\Sigma\left(\pi_{*} \widetilde{E}\right)$, by Corollary 5.3 .

Near $L_{0}=D$ on the other hand, restrict attention to a small neighborhood of $x_{0}$ isomorphic to $B\left(x_{0}, r_{0}\right)$ such that $\widetilde{B}\left(x_{0}, r_{0}\right):=\pi^{-1}\left(B\left(x_{0}, r_{0}\right)\right)$ is relatively compact in the support of $\sigma$, and choose holomorphic coordinates $\left(z^{0}, z^{1}\right)$ in the ball so that $\omega\left(x_{0}\right)$ is standard in these coordinates. If $\omega$ is the standard flat metric $\delta:=(i / 2) \partial \bar{\partial}|z|^{2}$ near $x_{0}$ and $\sigma=(i / 2 \pi) \partial \bar{\partial} \log |z|^{2}$ near $L_{0}$ then under the dilation $p_{\epsilon}: z \mapsto \sqrt{\epsilon} z$ the metric $\delta_{\epsilon}$ pulls-back to $\epsilon \delta_{1}$; in general, if $\omega$ and $\sigma$ are arbitrary, the pull-back of $\omega_{\epsilon}$ differs from $\epsilon\left(\pi^{*} \delta+\left.p_{0}^{*} \sigma\right|_{L_{0}}\right)$ by a term of order $\epsilon^{3 / 2}\left(\epsilon^{2}\right.$ if $d \omega=0, \sigma=(i / 2 \pi) \partial \bar{\partial} \log |z|^{2}$ and the coordinates are appropriately chosen), where $p_{0}: \widetilde{\mathbb{C}}^{2} \rightarrow L_{0}$ is the projection. For the purposes of this discussion, assume for simplicity that $\omega$ and $\sigma$ are standard near $x_{0}$ and $L_{0}$ respectively.

As $\epsilon \rightarrow 0$, the pull-backs $p_{\epsilon}^{*} \nabla_{\epsilon}$ to $B\left(x_{0}, r_{0} / \sqrt{\epsilon}\right)$ converge weakly off some finite set to a finite-action anti-self-dual connection on a bundle over $\widetilde{\mathbb{C}}^{2}$. As explained in [B4], after a gauge transformation this connection extends to a connection on a bundle over $\widetilde{\mathbb{P}}_{2}$ such that the connection is flat on the line 
$L_{\infty}$ at infinity and is anti-self-dual with respect to the (degenerate) metric $\delta_{1}$; collapsing $L_{\infty}$ to a point and flipping the orientation then gives a selfdual connection on a bundle over $\mathbb{C P}_{2}$, where self-duality is with respect to the Fubini-Study metric — that is, an instanton on $\mathbb{C P}_{2}([\mathrm{~B} 1])$. (The extension to the bundle on $\widetilde{\mathbb{P}}_{2}$ is obtained by applying Uhlenbeck's Removable Singularities theorem [U2] first to the one-point compactification of $\widetilde{\mathbb{C}}^{2}$.)

If $r=1$ and $\widetilde{E}=\mathcal{O}(-1)$, there is no bubbling of curvature and the sequence $p_{\epsilon}^{*} \nabla_{\epsilon}$ converges to the basic $\delta_{1}$-anti-self-dual connection on this line bundle over $\widetilde{\mathbb{P}}_{2}$. If $r>1$ and $\left.\widetilde{E}\right|_{L_{0}}=\oplus_{i=1}^{r} \mathcal{O}\left(a_{i}\right)$, it cannot be expected that the corresponding sequence $\left\{p_{\epsilon}^{*} \nabla_{\epsilon}\right\}$ should converge to the direct sum of the basic $\delta_{1}$-anti-self-dual connections on the line bundles $\mathcal{O}\left(a_{i}\right)$ in general, for if $\pi_{*} \widetilde{E}$ is stable the bounds on charge provided by Proposition 2.8 imply a bound on the charge of the limiting bundle which is strictly less $(1 / 2) \sum\left(a_{i}-\right.$ $a / r)^{2}$ if $\widetilde{E}$ does not split as a sum of line bundles in a neighborhood of $L_{0}$; $\left(a:=\sum a_{i}\right.$ as usual). However, when $\widetilde{E}$ is completely reducible near $L_{0}$, the anticipated result does hold:

Theorem 5.4. Let $\widetilde{X} \stackrel{\pi}{\rightarrow} X$ be the blowup of $X$ at $x_{0}$ with $L_{0}:=\pi^{-1}\left(x_{0}\right)$, and let $\widetilde{E}$ be an r-bundle on $\widetilde{X}$ with $\widetilde{E}=\oplus_{i} \mathcal{O}\left(a_{i}\right)$ in a neighborhood of $L_{0}$. If $\widetilde{E}$ admits an $\omega_{\epsilon}$-Hermitian-Einstein connection $\nabla_{\epsilon}$ for all $\epsilon>0$ suffciently small, then as $\epsilon \rightarrow 0$ the pull-back of $\nabla_{\epsilon}$ to $\widetilde{B}\left(x_{0}, r_{0} / \sqrt{\epsilon}\right)$ converges smoothly on compact subsets of $\widetilde{\mathbb{C}}^{2}$ (after suitable gauge transformations) to the direct sum of the standard anti-self-dual connections on $\mathcal{O}\left(a_{i}\right)$, where anti-self-duality is with respect to the metric $\pi^{*} \delta_{1}+\left.p_{0}^{*} \sigma\right|_{L_{0}}$ on $\widetilde{\mathbb{C}}^{2}$ in suitable holomorphic coordinates and where $p_{0}: \widetilde{\mathbb{C}}^{2} \rightarrow L_{0}$ is the projection.

Proof. Suppose first that $\pi_{*} \widetilde{E}$ is stable. Then by Lemma 2.2 of [B5] there is a family of automorphisms $g_{\epsilon}$ of the underlying topological bundle with $g_{\epsilon} \cdot \nabla_{\epsilon_{0}}=\nabla_{\epsilon}$ such that $\left|g_{\epsilon}\right|+\left|g_{\epsilon}^{-1}\right|$ is uniformly bounded on compact subsets of $X \backslash\left\{x_{0}\right\}$.

For each $i$, the bundle $\widetilde{E}\left(-a_{i}\right)$ has a nowhere-vanishing section in a neighborhood of $L_{0}$. Fix $i$ and let $s_{0}$ be a section of the topological bundle underlying $\widetilde{E}\left(-a_{i}\right)$ such that $s_{0}$ is non-vanishing in $\widetilde{B}\left(x_{0}, r_{0}\right)$ and $\bar{\partial}_{0} s_{0}=0$, where $\bar{\partial}_{0}$ is the $\bar{\partial}$-operator corresponding to $\nabla_{\epsilon_{0}}$. Then $s:=g_{\epsilon} s_{0}$ satisfies $\bar{\partial}_{\epsilon} s=0$, and $s$ is uniformly bounded on $\partial \widetilde{B}\left(x_{0}, r_{0}\right)$.

The curvature form $F\left(\nabla_{\epsilon}\right)$ of the Hermitian-Einstein connection on $\widetilde{E}\left(-a_{i}\right)$ satisfies $\omega_{\epsilon} \wedge i F\left(\nabla_{\epsilon}\right)=c_{\epsilon} \omega_{\epsilon}^{2} \mathbf{1}$, where $c_{\epsilon}=\left(\pi /\left(V-\epsilon^{2}\right)\right)\left[\mu\left(\pi_{*} \widetilde{E}, \omega\right)+\epsilon\left(a_{i}-\right.\right.$ $a / r)$ ]. The equation $\bar{\partial} s=0$ implies $\bar{\partial} \partial|s|^{2}=\langle\partial s, \partial s\rangle+\langle s, F s\rangle$, from which the equations

$$
\omega_{\epsilon} \wedge i \bar{\partial} \partial|s|^{2}+|\partial s|^{2} \omega_{\epsilon}^{2}=c_{\epsilon}|s|^{2} \omega_{\epsilon}^{2}
$$

and

$$
\omega_{\epsilon} \wedge i \bar{\partial} \partial \log |s|^{2}+|s|^{2}\left|\partial\left(s /|s|^{2}\right)\right|^{2} \omega_{\epsilon}^{2}=c_{\epsilon} \omega_{\epsilon}^{2}
$$


follow, where all derivatives of sections are with respect to $\nabla_{\epsilon}$, and the norms on 1 -forms are with respect to $\omega_{\epsilon}$.

Under the map $p_{\epsilon}: B\left(x_{0}, r_{0} / \sqrt{\epsilon}\right) \rightarrow B\left(x_{0}, r_{0}\right)$, pull back the data $\left(\nabla_{\epsilon}, s, \omega_{\epsilon}\right)$ to $\left(\nabla, s, \epsilon \omega_{1}\right)$ say, abusing notation slightly. Then the above equations respectively become

$$
\omega_{1} \wedge i \bar{\partial} \partial|s|^{2}+|\partial s|^{2} \omega_{1}^{2}=\epsilon c_{\epsilon}|s|^{2} \omega_{1}^{2}
$$

and

$$
\omega_{1} \wedge i \bar{\partial} \partial \log |s|^{2}+|s|^{2}\left|\partial\left(s /|s|^{2}\right)\right|^{2} \omega_{1}^{2}=\epsilon c_{\epsilon} \omega_{1}^{2}
$$

(where the norms on 1-forms are now those determined by $\omega_{1}$ ).

If $c_{\epsilon} \leq 0$ it follows from (5.6) and the Maximum Principle that $\log |s|^{2} \leq$ $\sup _{\partial \widetilde{B}} \log |s|^{2} \leq \log C_{0}$ for some fixed constant $C_{0}$ independent of $\epsilon$, where $\widetilde{B}:=\widetilde{B}\left(x_{0}, r_{0} / \sqrt{\epsilon}\right)$. If $c_{\epsilon}>0$ a similar bound still holds: For some constant $C_{1}>0$ independent of $\epsilon, \omega_{1} \wedge i \bar{\partial} \partial|z|^{2} \leq-C_{1} \omega_{1}^{2}$, so $\omega_{1} \wedge i \bar{\partial} \partial\left(\log |s|^{2}+\right.$ $\left.\epsilon c_{\epsilon}|z|^{2} / C_{1}\right) \leq 0$ giving $\log |s|^{2}+\epsilon c_{\epsilon}|z|^{2} / C_{1} \leq \sup _{\partial \widetilde{B}} \log |s|^{2}+\epsilon c_{\epsilon}|z|^{2} / C_{1} \leq$ $\log C_{0}+c_{\epsilon} r_{0}^{2} / C_{1}$ in $\widetilde{B}$; i.e., a uniform upper bound on $\log |s|^{2}$.

Since $\widetilde{E}$ splits as a direct sum in a neighborhood of $L_{0}$, it follows that there is a nowhere vanishing section $\lambda_{0}$ of $\widetilde{E}^{*}\left(a_{i}\right)$ in $\widetilde{B}\left(x_{0}, r_{0}\right)$ such that $\lambda_{0}\left(s_{0}\right) \equiv 1$. Then $\lambda:=g_{\epsilon}^{-1} \lambda_{0}$ satisfies $\bar{\partial}_{\epsilon} \lambda=0$ and is uniformly bounded on $\partial \widetilde{B}\left(x_{0}, r_{0}\right)$. Pulling back to $\widetilde{B}\left(x_{0}, r_{0} / \sqrt{\epsilon}\right)$ gives a section of the pull-back bundle (also denoted by $\lambda$ ) which is $\bar{\partial}$-closed with respect to the pulled-back connection, and which moreover satisfies $\lambda(s) \equiv 1$ in $\widetilde{B}$. The same argument as above applies to $\left(\lambda, p_{\epsilon}^{*} \widetilde{E}^{*}\left(a_{i}\right)\right)$ so $|\lambda|$ is uniformly bounded from above by a constant independent of $\epsilon$. Since $1=|\lambda(s)| \leq|\lambda||s|$, it follows that $|s|$ and $|\lambda|$ are also uniformly bounded above and away from 0 .

As $\epsilon \rightarrow 0$, the section $s=s(\epsilon)$ converges to a non-vanishing section of the limiting bundle on $\widetilde{\mathbb{C}}^{2}$; if there is any bubbling of curvature Hartogs' Theorem ensures that $s$ extends across the bad points. Moreover, once the limiting connection has been extended to a bundle $\widetilde{E}_{\infty}\left(-a_{i}\right)$ on $\widetilde{\mathbb{P}}_{2}$ so that it is flat on $L_{\infty}$, the section $s$ must also extend across $L_{\infty}$ by the Riemann Removable Singularities theorem, since $|s|$ is bounded; since the connection is flat on $L_{\infty}$, the restriction of $s$ to that line is covariantly constant.

The same argument applies to the limit of the sections $\lambda=\lambda(\epsilon)$, converging to a nowhere-vanishing section $\lambda$ of $\widetilde{E}_{\infty}^{*}\left(a_{i}\right)$ which satisfies $\lambda(s) \equiv 1$. Applying the argument for each $i=1, \ldots, r$ gives $r$ nowhere-vanishing sections $s_{i} \in H^{0}\left(\widetilde{\mathbb{P}}_{2}, \widetilde{E}_{\infty}\left(-a_{i}\right)\right)$ and $r$ nowhere-vanishing sections $\lambda_{i} \in H^{0}\left(\widetilde{\mathbb{P}}_{2}, \widetilde{E}^{*}\left(a_{i}\right)\right)$ such that $\lambda_{i}\left(s_{i}\right) \equiv 1$ for all $i$. Moreover, since $\left.\widetilde{E}\right|_{\widetilde{B}\left(x_{0}, r_{0}\right)}$ splits as a direct sum of line bundles, the sections $\lambda_{0, i}$ can be chosen initially to satisfy $H^{0}\left(\widetilde{B}\left(x_{0}, r_{0}\right), \mathcal{O}\left(a_{j}-a_{i}\right)\right) \ni \lambda_{0, j}\left(s_{0, i}\right) \equiv 0$ for $i \neq j$, so $\lambda_{i}\left(s_{j}\right) \equiv 0$ for $i \neq j$. Hence the sections $s_{1}, \ldots, s_{r}$ and $\lambda_{1}, \ldots, \lambda_{r}$ are linearly independent on $L_{\infty}$ 
and form a basis for $\widetilde{E}_{\infty}$ there (after trivialising $\left.\mathcal{O}(-1)\right|_{L_{\infty}}$ using a covariantly constant section of sup norm 1$)$.

From (5.5), the section $s_{j} \in H^{0}\left(\widetilde{\mathbb{P}}_{2}, \widetilde{E}_{\infty}\left(-a_{j}\right)\right)$ satisfies

$$
\omega_{1} \wedge \bar{\partial} \partial\left|s_{j}\right|^{2}+\left|\partial s_{j}\right|^{2} \omega_{1}^{2}=0
$$

(where now $\omega_{1}=\lim _{\epsilon \rightarrow 0} p_{\epsilon}^{*} \omega_{\epsilon} / \epsilon$ ), so by the Maximum Principle, the maximum of $\left|s_{j}\right|$ is attained on $L_{\infty}$, where it is constant, $c$ say. The same applies to $\lambda_{j}$, with $\left|\lambda_{j}\right|=d$ say on $L_{\infty}$. Since $\lambda_{j}\left(s_{i}\right)=0$ for $i \neq j$ it follows that on $L_{\infty}, 1=\left|\lambda_{j}\left(s_{j}\right)\right|=\left|\lambda_{j}\right|\left|s_{j}\right|=c d$. Thus on all of $\widetilde{\mathbb{P}}_{2}$, $1=\left|\lambda_{j}\left(s_{j}\right)\right| \leq\left|\lambda_{j}\right|\left|s_{j}\right| \leq c d=1$, implying equality everywhere. Consequently $c \geq\left|s_{j}\right|=c d /\left|\lambda_{j}\right| \geq c$, so $\left|s_{j}\right|$ and $\left|\lambda_{j}\right|$ are everywhere constant and (5.7) therefore implies that $s_{j}$ is covariantly constant. Hence the limiting connection inducing $\widetilde{E}_{\infty}$ is the direct sum of the standard $\omega_{1}$-anti-self-dual connections on the line bundles $\mathcal{O}\left(a_{i}\right), i=1, \ldots, r$.

Since $\widetilde{E}_{\infty}$ splits as a sum of the line bundles $\mathcal{O}\left(a_{i}\right)$, it follows $C\left(\widetilde{E}_{\infty}\right)=$ $(1 / 2) \sum_{i}\left(a_{i}-a / r\right)^{2}$. Since $r_{0}$ can be chosen so small that $\int_{\widetilde{X} \backslash \widetilde{B}\left(x_{0}, r_{0}\right)} \operatorname{tr}\left[F\left(\nabla_{\epsilon}\right)\right.$ $\left.-(1 / r) \operatorname{tr} F\left(\nabla_{\epsilon}\right) \mathbf{1}\right]^{2}$ differs from $8 \pi^{2} C\left(\left(\pi_{*} \widetilde{E}\right)^{* *}\right)$ by an arbitrarily small amount independent of $\epsilon$, it follows from (2.9) that

$$
\int_{\widetilde{B}\left(x_{0}, r_{0} / \sqrt{\epsilon}\right)} \operatorname{tr}\left[F\left(p_{\epsilon}^{*} \nabla_{\epsilon}\right)-(1 / r) \operatorname{tr} F\left(p_{\epsilon}^{*} \nabla_{\epsilon} \mathbf{1}\right)\right]^{2}
$$

differs from $8 \pi^{2}(1 / 2) \sum_{i}\left(a_{i}-a / r\right)^{2}$ by such an amount for arbitrary $\epsilon$; therefore there is no bubbling of charge in the sequence $\left\{p_{\epsilon}^{*} \nabla_{\epsilon}\right\}$ as $\epsilon \rightarrow 0$.

Suppose now that $\pi_{*} \widetilde{E}$ is not stable; by Proposition $5.2(\mathrm{a})$ this sheaf is semi-stable, and as $\epsilon \rightarrow 0$ Corollary 5.3 implies $\nabla_{\epsilon}$ converges on compact subsets of the complement of $\left\{x_{0}\right\} \cup B\left(\pi_{*} \widetilde{E}\right)$ to the Hermitian-Einstein connection on $\Sigma\left(\pi_{*} \widetilde{E}\right)$.

Choose a finite set of points $T$ disjoint from a $3 r_{0}$-neighborhood of $\left\{x_{0}\right\} \cup$ $B\left(\pi_{*} \widetilde{E}\right)$ and blowup $\widetilde{X}$ along $T$ to $\widetilde{X}^{\prime}$. Fix a metric on $\tilde{X}^{\prime}$ of the form $\omega_{\epsilon, \alpha}=\pi^{\prime *} \omega_{\epsilon}+\rho_{\alpha}$ for $\alpha=\delta_{0}(1, \ldots, 1)$, where $\operatorname{supp}\left(\rho_{\alpha} \cap \pi^{\prime-1} \widetilde{B}\left(x_{0}, 2 r_{0}\right)\right)=$ $\emptyset$, and fix a stabilization $E_{0}^{\prime}$ of $\Sigma\left(\pi_{*} \widetilde{E}\right)$. Similarly, for each $\epsilon$ construct a stabilization $\widetilde{E}_{\epsilon}^{\prime}$ of $\widetilde{E}$ on $\widetilde{X}^{\prime}$. The bundles $\widetilde{E}_{\epsilon}^{\prime}$ are determined by elements of $X_{x \in T} \mathbb{P}\left(\widetilde{E}_{x}^{*}\right)$ (where the vector space $\widetilde{E}_{x}$ is canonically determined by the connection $\left.\nabla_{\epsilon}\right)$. Since the connections $\nabla_{\epsilon}$ are converging strongly near $T$, in a neighborhood of $T$ there are converging sequences of holomorphic trivialisations, so the elements of $\times_{x \in T} \mathbb{P}\left(\widetilde{E}_{x}^{*}\right)$ can be chosen so that the bundles $\widetilde{E}_{\epsilon}^{\prime}$ are independent of $\epsilon$, all isomorphic to a fixed stabilization $\widetilde{E}^{\prime}$ of $\widetilde{E}$ and defined by a sequence of integrable connections $\nabla_{\epsilon}^{\prime}$ which agree with $\nabla_{\epsilon}$ outside a fixed neighborhood of $T$ and which converge smoothly in this neighborhood to a connection inducing $E_{0}^{\prime}$ there. 
Choose $\delta_{0}$ sufficiently small that $E_{0}^{\prime}$ is $\omega_{\alpha}$-stable. As in the proof of Proposition 3.4(d) there exists $\widetilde{A} \subset \widetilde{E}^{\prime}$ with torsion-free quotient such that $\widetilde{A}$ is $\omega_{\epsilon, \alpha}$-stable and maximises $\mu\left(\bullet, \omega_{\epsilon, \alpha}\right)$ for all $\epsilon>0$ sufficiently small. As $\epsilon \rightarrow 0$ the sequence $\left\{\nabla_{\epsilon}^{\prime}\right\}$ converges weakly off $B\left(\pi_{*} \widetilde{E}\right) \cup\left\{x_{0}\right\}$ to an integrable connection inducing $\widetilde{E}_{0}^{\prime}$; Lemma 2.2 of [B5] implies that there is a nonzero homomorphism $\left(\pi_{*} \widetilde{A}\right)^{* *} \rightarrow \widetilde{E}_{0}^{\prime}$ and since the latter is stable it follows $\mu\left(\pi_{*} \widetilde{A}, \omega_{\alpha}\right)<\mu\left(\widetilde{E}_{0}^{\prime}, \omega_{\alpha}\right)=\mu\left(\pi_{*} \widetilde{E}^{\prime}, \omega_{\alpha}\right)$. Therefore, for $\epsilon$ sufficiently small, $\mu\left(\widetilde{A}, \omega_{\epsilon, \alpha}\right)<\mu\left(\widetilde{E}^{\prime}, \omega_{\epsilon, \alpha}\right)$, implying $\widetilde{E}^{\prime}$ is $\omega_{\epsilon, \alpha}$-stable for all $\epsilon>0$ sufficiently small. Moreover, this same argument shows that $\pi_{*} \widetilde{E}^{\prime}$ must be $\omega_{\alpha}$-stable, otherwise there will exist stable $A \subset\left(\pi_{*} \widetilde{E}^{\prime}\right)^{* *}$ violating stability for which there is a non-zero holomorphic map $A \rightarrow E_{0}^{\prime}$.

Let $g_{\epsilon}$ be a complex automorphism of the topological bundle underlying $\widetilde{E}^{\prime}$ such that $g_{\epsilon} \cdot \nabla_{\epsilon}^{\prime}$ is $\omega_{\epsilon, \alpha}$-Hermitian-Einstein. If $X^{\prime}$ is the blowup of $X$ along $T$, then by weak compactness on $X^{\prime}$ there is a finite set $S^{\prime} \subset X^{\prime}$ such that this sequence of $\omega_{\epsilon, \alpha}$-Hermitian-Einstein connections has a subsequence converging weakly off $X^{\prime} \backslash\left(S^{\prime} \cup\left\{x_{0}\right\}\right)$ to define an $\omega_{\alpha}$-quasi-stable bundle $E_{1}^{\prime}$ on $X^{\prime}$. After rescaling $g_{\epsilon}$ if necessary, Lemma 2.2 of [B5] implies a subsequence converges in $C^{2}$ to a non-zero limit on the complement of a neighborhood of $B\left(\pi_{*} \widetilde{E}\right) \cup\left\{x_{0}\right\} \cup S^{\prime}$, which extends to a non-zero map $E_{1}^{\prime} \rightarrow E_{0}^{\prime}$. Since the two bundles have the same degree with respect to $\omega_{\alpha}$ and the latter is stable and the former is semi-stable, this map is an isomorphism. Hence $\left|g_{\epsilon}\right|+\left|g_{\epsilon}^{-1}\right|$ is uniformly bounded on compact subsets of the complement of $B\left(\pi_{*} \widetilde{E}\right) \cup\left\{x_{0}\right\} \cup S^{\prime}$; (in fact, $S^{\prime}=\emptyset$ ).

If $h_{\epsilon}:=g_{\epsilon}^{*} g_{\epsilon}$ then in a neighborhood of $L_{0}$ where $\rho_{\alpha} \equiv 0$ the equations $\omega_{\epsilon} \wedge i \bar{\partial} \partial \log \operatorname{tr} h_{\epsilon} \leq-\epsilon c_{\epsilon} \omega_{\epsilon}^{2}$ and $\omega_{\epsilon} \wedge i \bar{\partial} \partial \log \operatorname{tr} h_{\epsilon}^{-1} \leq \epsilon c_{\epsilon} \omega_{\epsilon}^{2}$ hold for some constant $c_{\epsilon}$ (converging to $\left.\pi \mu\left(\pi_{*} \widetilde{E}, \omega\right) \delta_{0}|T| / V^{2}\right)$. Since $\operatorname{tr} h_{\epsilon}$ and $\operatorname{tr} h_{\epsilon}^{-1}$ are uniformly bounded on the boundary of this neighborhood, the same argument as was used for the sections $s$ in the case that $\pi_{*} \widetilde{E}$ is stable shows that $\operatorname{tr} h_{\epsilon}$ and $\operatorname{tr} h_{\epsilon}^{-1}$ are uniformly bounded inside the neighborhood also. Hence the two sequences of connections $\left\{g_{\epsilon} \cdot \nabla_{\epsilon}\right\}$ and $\left\{\nabla_{\epsilon}\right\}$ and their pullbacks to $\widetilde{B}\left(x_{0}, r_{0} / \sqrt{\epsilon}\right)$ have the same weak convergence and weak limits, by Lemma 2.1 of $[\mathbf{B 5}]$. Since $\pi_{*} \widetilde{E}^{\prime}$ is $\omega_{\alpha}$-stable, the proof of the theorem is complete.

If $\widetilde{E}$ does not split as a direct sum of line bundles in a neighborhood of $L_{0}$, the sequence $\left\{\nabla_{\epsilon}\right\}$ still converges strongly on compact subsets of $\widetilde{\mathbb{P}}_{2} \backslash\left(L_{0} \cup L_{\infty}\right)$ to a finite action connection which is $\omega_{1}$-anti-self-dual and which extends to $\widetilde{\mathbb{P}}_{2}$ to define a bundle $\widetilde{E}_{\infty}$ which is trivial in neighborhood of $L_{\infty}$ - the arguments are essentially the same as those given above, using the fact that $\pi_{*} \widetilde{E}(-k)$ and $\pi_{*} \widetilde{E}^{*}(-k)$ will be locally free for sufficiently large 
$k$ to obtain bounded sections which have uniform lower bounds on norms away from $L_{0}$.

\section{Compactification of moduli spaces.}

Let $X$ be a compact complex surface equipped with a positive $\bar{\partial} \partial$-closed $(1,1)$-form $\omega$, and let $\left\{E_{i}\right\}$ be a sequence of stable bundles on $X$ converging weakly off $S$ to a quasi-stable bundle $E$. Blow up $X$ along $S$ to $\widetilde{X} \stackrel{\pi}{\rightarrow} X$ and pull back the sequence $\left\{E_{i}\right\}$. Assume temporarily that every bundle in the pulled-back sequence is stable with respect to the same metric $\omega_{\alpha}$ for some suitable $\alpha$ sufficiently small - this will be true if either $b_{1}(X)$ is even (by Proposition 3.5) or if $E$ is stable. (If $\widetilde{A}_{i} \subset \pi^{*} E_{i}$ maximises $\mu\left(\bullet, \omega_{\epsilon \alpha}\right)$ for all $\epsilon>0$ sufficiently small, then $\pi_{*} \widetilde{A}_{i}$ is semi-stable. If $\nu_{\pi^{*} E_{i}}\left(\widetilde{A}_{i}, \omega_{\epsilon_{i} \alpha}\right)=0$ for $\epsilon_{i} \rightarrow 0$, then as in $\S 3$, the ranks, first Chern classes, charges, and degrees of $\pi_{*} \widetilde{A}_{i}$ are all uniformly bounded, and the same holds for any stable $A_{i}^{\prime} \subset \pi_{*} \widetilde{A}_{i}$ of the same degree. Passing to a subsequence, taking a weak limit and applying semi-continuity of cohomology then yields a semi-stable bundle $A$ with $\operatorname{rank}(A)<\operatorname{rank}(E), \mu(A)=\mu(E)$ and a non-zero holomorphic map $A \rightarrow E$, contradicting stability of $E$.)

By weak compactness on $\widetilde{X}$ the new sequence converges weakly off some finite set $\widetilde{S} \subset \widetilde{X}$ to a semi-stable bundle $\widetilde{E}$ and by semi-continuity of cohomology there is a non-zero holomorphic map $\left(\pi_{*} \widetilde{E}\right)^{* *} \rightarrow E$. If either is stable, then this map is an isomorphism and it follows from Lemma 2.1 of [B5] that $\widetilde{S} \subset \pi^{-1} S$; moreover $C(\widetilde{E}) \geq C(E)$ by Proposition 2.8.

This argument suggests that there is another compactification of moduli spaces tied more closely to the complex analysis, distinct from the more usual ones ([Gie], [M]): If $\widetilde{X} \stackrel{\pi}{\rightarrow} X$ is a blowup of $X$ at $x_{0}$, sequences of stable bundles $\widetilde{E}_{i}$ on $\widetilde{X}$ are easily constructed such that each is trivial on the exceptional line and such that the sequence converges smoothly on $\widetilde{X}$ to a bundle $\widetilde{E}$ which is stable but which is now non-trivial on the exceptional line. Thus the theorems of Uhlenbeck [U1], [U2] on compactness and removability of singularities in the space of Yang-Mills connections are reinterpreted as the well-known phenomenon of jumping of holomorphic structures. It is natural to conjecture that this is the only type of catastrophe which can occur, and that, given a degenerating sequence of Hermitian-Einstein connections on a bundle over $X$, after a finite number blowups and pull-backs, a strongly convergent subsequence can be found. Unfortunately, there is no guarantee that after blowing-up and pulling-back, the new sequence of Hermitian-Einstein connections will not bubble precisely the same amount of charge as the original and that iterating the process will eventually lead to a convergent subsequence. Indeed, by a process of diagonalisation, sequences of connections can be constructed for which this blowing-up and 
pulling-back procedure will not terminate after finitely many steps. Despite this, provided that some flexibility is introduced into the blowing-up process, a form of compactness does hold, as is indicated by the following result proved in $[\mathbf{B 5}]$ :

Theorem 6.1. Let $X$ be a compact complex surface equipped with a positive $\bar{\partial} \partial$-closed $(1,1)$-form $\omega$. Let $\left\{A_{i}\right\}$ be a sequence of Hermitian-Einstein connections on a fixed unitary bundle $E_{\text {top }}$ of rankr over $X$ such that the corresponding holomorphic bundles $E_{i}$ are stable and are of uniformly bounded degree. Suppose that $E_{i}$ converges weakly to $E$ off the finite set $S \subset X$ consisting of $|S|$ points. Then for some subsequence $\left\{E_{i_{j}}\right\} \subset\left\{E_{i}\right\}$ :

1. There is a sequence of blowups $\widetilde{X}_{i_{j}}$ of $X$ consisting of at most $2 C\left(E_{\mathrm{top}}\right)$ $-2 C(E)-|S|$ individual blowups converging to a blowup $\widetilde{X} \stackrel{\pi}{\rightarrow} X$ of $X$ with exceptional divisor $\pi^{-1}(S)$;

2. There are complex automorphisms $g_{i_{j}}$ of $\pi_{i_{j}}^{*} E_{\text {top }}$ such that $\left|g_{i_{j}}\right|+\left|g_{i_{j}}^{-1}\right|$ is uniformly bounded on compact subsets of $\widetilde{X} \backslash \pi^{-1}(S)$ and $\left\{g_{i_{j}}\right\}$ is converging uniformly in $C^{3}$ on such subsets;

3. $\left\{g_{i_{j}} \cdot\left(\pi_{i_{j}}^{*} A_{i_{j}}\right)\right\}$ converges uniformly in $C^{2}$ to a smooth connection on $\pi^{*} E_{\text {top }}$ over $\widetilde{X}$ which defines a holomorphic bundle $\widetilde{E}$ such that $\left(\pi_{*} \widetilde{E}\right)^{* *}$ $=E$;

4. If $E$ is stable then for any suitable $\alpha$ with $|\alpha|$ sufficiently small, the connections $g_{i_{j}} \cdot\left(\pi_{i_{j}}^{*} A_{i_{j}}\right)$ can be taken to be $\left(\pi_{i_{j}}^{*} \omega+\rho_{\alpha}\right)$-HermitianEinstein, and $\widetilde{E}$ is $\omega_{\alpha}$-stable.

If $E$ is not stable, but $b_{1}(X)$ is even and $\operatorname{rank}\left(E_{\mathrm{top}}\right)=2$ there is sequence of blowups $\left\{X_{i_{j}}\right\}$ consisting of at most $2 C\left(E_{\mathrm{top}}\right)-2 C(E)-1$ individual blowups converging to a blowup $\widetilde{X}$ such that, for some suitable $\alpha, \pi_{i}^{*} E_{i}$ is $\omega_{\epsilon \alpha}$-stable for all $\epsilon \in(0,1]$ and the subsequence $\left\{\pi_{i_{j}}^{*} E_{i_{j}}\right\}$ converges strongly to a bundle $\widetilde{E}$ on $\widetilde{X}$, stable with respect to $\omega_{\epsilon \alpha}$-stable for all $\epsilon \in(0,1]$ (assuming here that $|S|>0)$.

The convergence of a sequence of blowups should be interpreted as the convergence of a sequence of integrable complex structures on the same underlying smooth manifold $X \# n \overline{\mathbb{P}}_{2}$ endowed with a fixed Riemannian metric. In the current setting, these complex structures are isomorphic on the complement of a fixed open set with strictly pseudoconvex boundary.

A priori, there is no reason why the last statement of the theorem cannot be extended to bundles of arbitrary rank, but as yet a proof is lacking. The complications arise, as always, from the presence of bundles which are semi-stable but not stable.

Theorem 6.1 suggests a variety of different compactifications for moduli of stable bundles - for example, adding torsion-free semi-stable sheaves which 
are direct images of stable bundles on blowups is an obvious candidate. One other which has some interesting properties is described below.

Let $E_{\text {top }}$ be a unitary $r$-bundle over $(X, \omega)$ and let $\mathcal{M}\left(X, E_{\text {top }}\right)$ denote the space of isomorphism classes of quasi-stable holomorphic structures on $E_{\text {top. }}$ Consider the set of pairs $(\widetilde{X}, \widetilde{E})$ where

1. $\widetilde{X}$ is a blowup of $X$;

2. $\widetilde{E}$ is a holomorphic bundle on $\widetilde{X}$ topologically isomorphic to $\pi^{*} E_{\mathrm{top}}$ such that $\pi_{*} \widetilde{E}$ is semi-stable;

3. $\widetilde{E}$ is $\omega_{\alpha}$-quasi-stable for all suitable $\alpha$ in an open set of such;

4. If $b_{1}(X)$ is odd, $\operatorname{deg}\left(\pi_{*} \widetilde{E}, \omega\right)=0$.

Note that the third condition implies that if $\widetilde{E}$ is not stable, then it is a direct sum of stable bundles each of which is topologically trivial on the exceptional divisor. Note also that the requirement that $\pi_{*} \widetilde{E}$ be semi-stable implies that $\widetilde{E}$ is $\omega_{\epsilon \alpha^{-}}$(quasi-)stable for all $\epsilon \in(0,1]$ if $\widetilde{E}$ is $\omega_{\alpha^{-}}$(quasi-)stable.

If $b_{1}(X)$ is odd, it follows from the proof of Proposition 2 in [B3] that for any $t \in \mathbb{R}$ there is a line bundle $L_{t}$ on $X$ with $c_{1}\left(L_{t}\right)=0$ and $\operatorname{deg}\left(L_{t}, \omega\right)=t$, so the fourth condition simply prevents this type of non-compactness.

On the set of pairs $(\widetilde{X}, \widetilde{E})$ satisfying the above conditions, define an equivalence relation $\sim$ by setting $\left(\widetilde{X}_{1}, \widetilde{E}_{2}\right) \sim\left(\widetilde{X}_{2}, \widetilde{E}_{2}\right)$ if there is a joint blowup $\widetilde{X}_{12}$ such that $\pi_{1}^{*} \widetilde{E}_{1} \simeq \pi_{2}^{*} \widetilde{E}_{2}$ on $\widetilde{X}_{12}$, and let $\overline{\mathcal{M}}=\overline{\mathcal{M}}\left(X, E_{\text {top }}\right)$ denote the set of equivalence classes.

A sequence $\left\{\left[\left(\widetilde{X}_{i}, \widetilde{E}_{i}\right)\right]\right\} \subset \overline{\mathcal{M}}$ is decreed to converge to $[(\widetilde{X}, \widetilde{E})]$ if and only if $\left[\left(\widetilde{X}_{i}, \widetilde{E}_{i}\right)\right]$ can be represented by a sequence of blowups $\widetilde{X}_{i}$ converging to $\widetilde{X}$ with $\omega_{\alpha}$-quasi-stable bundles $\widetilde{E}_{i}$ on $\widetilde{X}_{i}$ converging strongly to $\widetilde{E}$ on $\widetilde{X}$. That is, the complex surfaces $\widetilde{X}_{i}$ are all diffeomorphic to $\widetilde{X}$ with the complex structures isomorphic outside a neighbourhood of the exceptional divisor and converging in $\mathcal{C}^{k}$ with respect to some fixed metric on $\widetilde{X}$, and the corresponding sequence of $\omega_{\alpha}$-Hermitian-Einstein connections, viewed as a sequence of unitary connections on the same topological bundle over a fixed 4-manifold, is converging in $\mathcal{C}^{k}$ to the Hermitian-Einstein connection inducing $\widetilde{E}$; here $k$ is some large fixed integer. A topology is defined in the usual way by deeming sets to be closed if and only if they contain all of their limit points.

Given $[(\widetilde{X}, \widetilde{E})] \in \overline{\mathcal{M}}$ with $\widetilde{E}$ quasi-stable with respect to $\omega_{\alpha^{\prime}}$ for all $\alpha^{\prime}$ in a neighborhood of $\alpha$, let $U\left(A_{0}, \epsilon_{1}, \epsilon_{2}\right)$ be the set of unitary connections on $\pi^{*} E_{\text {top }}$ of the form $A_{0}+a$ where $\|a\|_{C^{k}\left(\omega_{\alpha}\right)}<\epsilon_{1}$ and where $A_{0}+a$ has curvature of type $(1,1)$ with respect to an integrable complex structure $J$ on the smooth manifold underlying $\widetilde{X}$, with $J$ within $\epsilon_{2}$ in $\mathcal{C}^{k}$ of that defining $\widetilde{X}$. The connection $A_{0}+a$ is moreover required to be Hermitian-Einstein with respect to the hermitian metric corresponding to $\omega_{\alpha}$, defining a holomorphic 
bundle which is $\omega_{\alpha^{\prime}}$-quasi-stable for all $\alpha^{\prime}$ in a neighborhood of $\alpha$. It is not hard to verify that the image in $\overline{\mathcal{M}}$ of a set of this form is open and that every open neighborhood of $[(\widetilde{X}, \widetilde{E})]$ in $\overline{\mathcal{M}}$ contains the image of such a set. Hence the collection of (the images in $\overline{\mathcal{M}}$ of) such sets forms a base for the topology on $\overline{\mathcal{M}}$, from which it follows that this topology is second countable.

Theorem 6.1 implies that at least under certain circumstances, every sequence in $\mathcal{M}$ has a subsequence converging in $\overline{\mathcal{M}}$. To attempt to use this to construct a compactification of $\mathcal{M}$ clearly requires a bound on the number of blowups required to represent classes in $\overline{\mathcal{M}}$, a bound which is provided by Proposition 6.3 below.

Lemma 6.2. For any $r_{0}, C_{0}$ there exists $n=n\left(r_{0}, C_{0}, \omega\right)$ such that any semi-stable bundle $E$ of rank $\leq r_{0}$ and charge $\leq C_{0}$ satisfies $h^{2}\left(X\right.$, End $\left._{0} E\right)$ $\leq n$.

Proof. If not, there exists a sequence $\left\{E_{i}\right\}$ of semi-stable bundles of bounded rank and charge such that $h^{2}\left(X\right.$, End $\left._{0} E_{i}\right) \rightarrow \infty$. It can be assumed without loss of generality that each $E_{i}$ is quasi-stable since it follows easily by induction on rank that $h^{2}\left(\operatorname{End}_{0} E\right) \leq h^{2}\left(\operatorname{End}_{0} \Sigma(E)\right)$ for any semi-stable $E$. The bundles End $_{0} E_{i}$ have uniformly bounded ranks $\left(\leq r_{0}^{2}-1\right)$ and charges $\left(\leq 2 r_{0} C_{0}\right)$ with trivial determinants, and each is also quasi-stable. By passing to a subsequence if necessary, it can be assumed that End ${ }_{0} E_{i}$ is converging weakly to a quasi-stable bundle $B$ off some finite set $S \subset X$. Passing to another subsequence and using Theorem 6.1, there is a sequence of blowups $\left\{\widetilde{X}_{i}\right\}$ converging to a blowup $\widetilde{X}$ such that a sequence of smooth integrable connections representing $\pi_{i}^{*}$ End $_{0} E_{i}$ converges strongly in $C^{2}$ to define a bundle $\widetilde{B}$ on $\widetilde{X}$ with $\left(\pi_{*} \widetilde{B}\right)^{* *}=B$. Then $h^{2}\left(\widetilde{X}_{i}, \pi_{i}^{*} \operatorname{End}_{0} E_{i}\right)=$ $h^{0}\left(\widetilde{X}_{i},\left(\pi_{i}^{*} \operatorname{End}_{0} E_{i}\right) \otimes K_{\widetilde{X}_{i}}\right)=h^{0}\left(X,\left(\operatorname{End}_{0} E_{i}\right) \otimes K_{X}\right)=h^{2}\left(X, \operatorname{End}_{0} E_{i}\right) \rightarrow$ $\infty$, contradicting the fact that $h^{2}\left(\widetilde{X}_{i}, \pi_{i}^{*} \operatorname{End}_{0} E_{i}\right) \leq h^{2}(\widetilde{X}, \widetilde{B})$ for $i$ sufficiently large, by standard semi-continuity of cohomology.

Proposition 6.3. There is an integer $N=N\left(X, \omega, E_{\text {top }}\right)$ such that every class in $\overline{\mathcal{M}}$ can be represented by a bundle on a blowup consisting of at most $N$ individual blowups. If $\operatorname{deg}\left(K_{X}, \omega\right)<0$ then $N \leq 2 C\left(E_{\text {top }}\right)-1$.

Proof. Suppose $[(\widetilde{X}, \widetilde{E})] \in \overline{\mathcal{M}}$ and let $\widetilde{S} \subset \widetilde{X}$ be the exceptional divisor. Assume initially that $\pi_{*} \widetilde{E}$ on $X$ is stable and moreover that $H^{2}(\widetilde{X}$, $\left.\left(\operatorname{End}_{0} \widetilde{E}\right)(-\widetilde{S})\right)=0$. As discussed in $\S 4$, the latter assumption implies that $\widetilde{E}$ has arbitrarily small deformations which are trivial on every irreducible component of $\widetilde{S}$ and the former assumption implies that $\widetilde{E}$ is $\omega_{\alpha^{-}}$-stable for sufficiently small suitable $\alpha$. It follows that there is a sequence of stable bundles $\left\{E_{i}\right\}$ on $X$ such that $\pi^{*} E_{i}$ is converging strongly on $\widetilde{X}$ to $\widetilde{E}$ with respect to $\omega_{\alpha}$ for any such $\alpha$. 
The sequence $\left\{E_{i}\right\}$ on $X$ converges weakly off some finite set $T \subset X$ to yield a quasi-stable bundle $E$, and by Lemma 2.2 of [B5] (semi-continuity of cohomology) there is a non-zero holomorphic map $E \rightarrow\left(\pi_{*} \widetilde{E}\right)^{* *}$. By stability of the latter bundle, this map must be an isomorphism, and by Lemma 2.1 of [B5] and the discussion following Lemma 2.2 of [B5] it then follows that $T \subset \pi(\widetilde{S})$.

By Theorem 6.1 , there is a sequence $\left\{\widetilde{X}_{i}\right\}$ of blowups of $X$ converging to a blowup $\widetilde{X}_{1}$ of $X$ with each consisting of at most $2 C(\widetilde{E})-2 C(E)-|T|$ individual blowups such that $\pi_{i}^{*} E_{i}$ is converging strongly to a stable bundle $\widetilde{E}_{1}$ on $\widetilde{X}_{1}$, with $\left(\pi_{1 *} \widetilde{E}_{1}\right)^{* *}=E$. Pulling back to a joint blowup of $\widetilde{X}_{i}$ and $\widetilde{X}$ and picking a subsequence of these blowups which converges to a joint blowup $\widetilde{X}_{2}$ of $\widetilde{X}$ and $\widetilde{X}_{1}$, the stability of $\widetilde{E}$ and of $\pi_{2}^{*} \widetilde{E}_{1}$ together with semicontinuity of cohomology shows that $\left(\widetilde{X}_{1}, \widetilde{E}_{1}\right) \sim(\widetilde{X}, \widetilde{E})$. The desired result is therefore proved in this case.

If $\pi_{*} \widetilde{E}$ is still assumed to be stable but it is no longer assumed that $H^{2}\left(\widetilde{X},\left(\operatorname{End}_{0} \widetilde{E}\right)(-\widetilde{S})\right)=0$, pick $n=h^{2}\left(\widetilde{E},\left(\operatorname{End}_{0} \widetilde{E}\right)(-\widetilde{S})\right)$ points disjoint from $\widetilde{S}$ and blowup $\widetilde{X}$ to $\widetilde{X}_{1}$ at these points. Now apply Proposition 4.4 to construct a modification $\widetilde{E}_{1}$ of $\widetilde{E}$ on $\widetilde{X}_{1}$ which has the property that $H^{2}\left(\widetilde{X}_{1},\left(\operatorname{End}_{0} \widetilde{E}_{1}\right)(-\widetilde{S})\right)=0$. Applying the result of the previous paragraph, $\widetilde{E}_{1}$ can be represented by a bundle on a blowup of $X$ consisting of a finite number of blowups, where the number is bounded by a function of $C(\widetilde{E})$ and $r$ (using Lemma 6.2). Blowing down the $n$ lines in the blowup of $\widetilde{X}_{1}$ used for the construction of $\widetilde{E}_{1}$ and taking direct images followed by double-duals then gives the same result as before; (recall that the $n$ exceptional lines for the blowup $\widetilde{X}_{1} \rightarrow \widetilde{X}$ are disjoint from $\left.\widetilde{S}\right)$.

Finally, if it is no longer assumed that $\pi_{*} \widetilde{E}$ be stable, first stabilize $\pi_{*} \widetilde{E}$ using a set of $r^{2}+1$ points disjoint from $\pi(\widetilde{S})$ and apply the same method as in the previous paragraph.

If $\operatorname{deg}\left(K_{X}, \omega\right)<0$ then $\widetilde{E}$ has arbitrarily small deformations which are trivial on every component of the exceptional divisor $\widetilde{S}$, so only the second type of stabilization is required for any $\widetilde{E}$ in this case. Since the new bundle representing the stabilized bundle $\widetilde{E}_{1}$ must be non-trivial on each of the $r^{2}+1$ lines introduced for the stabilization, the count on the number of blowups required to obtain the new representative for $[(\widetilde{X}, \widetilde{E})]$ obtained from the first statement of Theorem 6.1 is $2 C\left(\widetilde{E}_{1}\right)-2 C\left(\pi_{*} \widetilde{E}_{1}\right)-|\pi(\widetilde{S})| \leq$ $2 C(\widetilde{E})-2 C\left(\pi_{*} \widetilde{E}\right)-|\pi(\widetilde{S})| \leq 2 C(\widetilde{E})-1$.

\section{Theorem 6.4.}

1. $\mathcal{M} \subset \overline{\mathcal{M}}$ is an open set which is dense if $\operatorname{deg}\left(K_{X}, \omega\right)<0$. Moreover the subspace corresponding to the indecomposable bundles $\overline{\mathcal{M}}_{\text {stable }} \subset \overline{\mathcal{M}}$ is smooth in this case. 
2. If $b_{1}(X)$ is even and $\operatorname{rank}\left(E_{\text {top }}\right)=2$ then $\overline{\mathcal{M}}$ is compact.

3. If every semi-stable bundle $E$ on $X$ satisfying $\operatorname{rank}(E)=\operatorname{rank}\left(E_{\mathrm{top}}\right)$, $c_{1}(E)=c_{1}\left(E_{\mathrm{top}}\right)$, and $C(E) \leq C\left(E_{\mathrm{top}}\right)\left(\right.$ and $\operatorname{deg}(E, \omega)=0$ if $b_{1}(X)$ is odd) is stable then $\overline{\mathcal{M}}$ is a compact Hausdorff space.

Note that if $b_{1}(X)$ is even and if $c_{1}\left(E_{\mathrm{top}}\right)$ has non-zero image in $H^{2}(X, \mathbb{R})$ and its image in $H^{2}\left(X, \mathbb{Z}_{r}\right)$ is not a torsion class (where $r=\operatorname{rank}\left(E_{\text {top }}\right)$ ), the hypothesis in the third statement holds for generic $\omega$. Even if $c_{1}\left(E_{\mathrm{top}}\right)=0$, by fixing a base point $x_{0} \in X$ and blowing up at this point, the $\mathbb{P}_{r-1}$-bundle over $\mathcal{M}_{\text {stable }} \subset \mathcal{M}$ with fibre $\mathbb{P}\left(E_{x_{0}}\right)$ at $E \in \mathcal{M}_{\text {stable }}$ is isomorphic to the space of $\omega_{\epsilon}$-stable bundles on $\tilde{X}$ restricting to $\mathcal{O}(-1) \oplus \mathcal{O}^{r-1}$ on $\pi^{-1}\left(x_{0}\right)$ with direct image topologically isomorphic to $E_{\text {top }}$.

\section{Proof of Theorem 6.4 .}

1. If $\left\{\left(\widetilde{X}_{i}, \widetilde{E}_{i}\right)\right\} \subset \overline{\mathcal{M}} \backslash \mathcal{M}$ is converging to $(\widetilde{X}, \widetilde{E}) \in \mathcal{M}$ (abusing notation in the obvious way), there is an irreducible component of the exceptional divisor in $\widetilde{X}_{i_{j}}$ to which $\widetilde{E}_{i_{j}}$ restricts non-trivially for every $i_{j}$ in some subsequence, and by passing to another subsequence if necessary, it can be assumed that these components $L_{i_{j}}$ are converging to some irreducible component $L$ of the exceptional divisor in $\widetilde{X}$. Then $H^{0}\left(L_{i_{j}}, \widetilde{E}_{i_{j}}(-1)\right) \neq 0$ so by semi-continuity of cohomology it follows $H^{0}(L, \widetilde{E}(-1)) \neq 0$, implying $\widetilde{E} \in \overline{\mathcal{M}} \backslash \mathcal{M}$ and hence that $\mathcal{M}$ is an open set.

If $\operatorname{deg}\left(K_{X}, \omega\right)<0$ then $\operatorname{deg}\left(\operatorname{End}_{0} \widetilde{E} \otimes K_{\widetilde{X}}, \omega_{\alpha}\right)<0$ for any bundle $\widetilde{E}$ on a blowup $\widetilde{X}$ stable with respect to $\omega_{\alpha}$ for $|\alpha|$ small enough, so $H^{2}\left(\widetilde{X}, \operatorname{End}_{0} \widetilde{E}\right)$ $=H^{0}\left(\widetilde{X},\left(\text { End }_{0} \widetilde{E}\right) \otimes K_{\widetilde{X}}\right)^{*}=0$ for any such bundle, implying that the infinitesimal deformations of $\widetilde{E}$ are unobstructed. If, in addition, $H^{0}\left(\widetilde{X}, \operatorname{End}_{0} \widetilde{E}\right)$ $=0$ (i.e., $\widetilde{E}$ is stable given that it is quasi-stable), it is a standard fact that the space of deformations of $\widetilde{E}$ is smooth near $\widetilde{E}$.

2, 3. Suppose $\left\{\left[\left(\widetilde{X}_{i}, \widetilde{E}_{i}\right)\right]\right\} \subset \overline{\mathcal{M}}$. By Proposition 6.3 , it can be assumed that for each $i, c_{2}\left(\widetilde{X}_{i}\right) \leq c_{2}(X)+N$ and by passing to a subsequence, it can then be assumed that $c_{2}\left(\widetilde{X}_{i}\right)=c_{2}(X)+n$ is constant. It is easy to see (by induction on $n$ for example), that there is a subsequence such that $\widetilde{X}_{i_{j}}$ converges to a blowup $\widetilde{X}$ of $X$.

There are finitely many classes in $c \in H^{2}(X, \mathbb{Z})^{\perp} \subset H^{2}(\widetilde{X}, \mathbb{Z})$ satisfying $-c \cdot c \leq r^{3} C\left(E_{\text {top }}\right)$ and therefore only finitely different classes of metrics on $\widetilde{X}_{i}$ of the form $\omega_{i, \alpha}:=\pi_{i}^{*} \omega+\rho_{\alpha}$ with respect to which $\widetilde{E}_{i}$ can be quasistable for all $\alpha$ in an open set. By passing to a subsequence, one such class of metrics can be fixed, and then for some new subsequence a corresponding sequence of metrics can be found converging to a non-degenerate metric $\omega_{\alpha}$ on $\widetilde{X}$. 
The $\omega_{i, \alpha}$-Hermitian-Einstein connection on $\widetilde{E}_{i}$ has curvature bounded in $L^{2}\left(\omega_{i, \alpha}\right)$, and hence in $L^{2}\left(\omega_{\alpha}\right)$ since the metrics are converging and hence compare uniformly. Regarding the sequence of connections as a sequence of unitary connections on the fixed bundle $\pi^{*} E_{\text {top }}$ over the smooth Riemannian manifold $\left(X \# n \overline{\mathbb{P}}_{2}, \omega_{\alpha}\right)$ with uniformly $L^{2}$-bounded curvature, Uhlenbeck's weak compactness result implies that after passing to another subsequence there is a finite set of points where the curvature is bubbling, with the sequence converging (after gauge transformations) weakly in $L_{1, \text { loc }}^{2}$ on the complement. Convergence of the sequence of metrics $\left\{\omega_{i, \alpha}\right\}$ and ellipticity of the Hermitian-Einstein equations imply that for some subsequence, this convergence can be bootstrapped to uniform convergence in $C^{k}$ on compact subsets of the complement of the bad set, converging weakly to an $\omega_{\alpha}$-quasistable limit $\widetilde{E}$ on $\widetilde{X}$.

By the same arguments as in the proof of Theorem 1.3 of [B5], after passing to another subsequence if necessary, there is a further sequence of blowups $\left\{\widetilde{X}_{i}^{\prime}\right\}$ such that $\widetilde{X}_{i}^{\prime}$ is a blowup of $\widetilde{X}_{i}$ consisting of at most $2\left(C\left(E_{\text {top }}\right)-C(\widetilde{E})\right)-1$ blowups, $\pi_{i}^{\prime *} \widetilde{E}_{i}$ is $\omega_{i, \alpha, \beta}$-quasi-stable and some sequence of smooth integrable connections inducing $\pi_{i}^{\prime *} \widetilde{E}_{i}$ converges strongly on $\widetilde{X}^{\prime}$ to a bundle $\widetilde{E}^{\prime}$ satisfying $\left(\pi_{*}^{\prime} \widetilde{E}^{\prime}\right)^{* *}=\widetilde{E}$.

Under the hypotheses of the third statement of the theorem, $\pi_{*} \widetilde{E}$ must be stable and therefore so too is $\widetilde{E}$ with respect to $\omega_{\alpha}$ for any suitable $\alpha$ with $|\alpha|$ sufficiently small by Proposition 3.4. Hence so too is $\widetilde{E}^{\prime}$ if $|\beta|$ is small enough, proving that $\overline{\mathcal{M}}$ is sequentially compact (and hence compact, by second countability of the topology) in this case. Furthermore, if a sequence $\left\{\left(\widetilde{X}_{1, i}, \widetilde{E}_{1, i}\right)\right\} \subset \overline{\mathcal{M}}$ converges to $\left(\widetilde{X}_{1}, \widetilde{E}_{1}\right)$ and $\left(\widetilde{X}_{1, i}, \widetilde{E}_{1, i}\right) \sim\left(\widetilde{X}_{2, i}, \widetilde{E}_{2, i}\right)$ with $\left\{\left(\widetilde{X}_{2, i}, \widetilde{E}_{2, i}\right)\right\}$ converging to $\left(\widetilde{X}_{2}, \widetilde{E}_{2}\right)$, the sequence of joint blowups $\widetilde{X}_{12, i}$ has a subsequence converging to a joint blowup $\widetilde{X}_{12}$ of $\widetilde{X}_{1}$ and $\widetilde{X}_{2}$, and $\pi_{1}^{*} \widetilde{E}_{1}$, $\pi_{2}^{*} \widetilde{E}_{2}$ must both be stable with respect to any metric on $\widetilde{X}_{12}$ of the form $\omega_{\alpha, \beta}$ for $|\alpha|+|\beta|$ sufficiently small. Since semi-continuity of cohomology gives a non-zero holomorphic map between these two pull-backs, this map must be an isomorphism. Thus $\left(\widetilde{X}_{1}, \widetilde{E}_{1}\right) \sim\left(\widetilde{X}_{2}, \widetilde{E}_{2}\right)$ and therefore the topology on $\overline{\mathcal{M}}$ is Hausdorff.

If $b_{1}(X)$ is even and $\operatorname{rank}\left(E_{\mathrm{top}}\right)=2$, the same arguments as given in $\S 7$ of [B5] to prove Theorem 1.4 of that reference (i.e., the last statement of Theorem 6.1 above) can be repeated and yield the same conclusion as that above; i.e., that $\overline{\mathcal{M}}$ is compact in this case.

\section{References}

[BS] C. Bănică and O. Stănăsilă, Algebraic methods in the global theory of complex spaces, Wiley, London, New York, 1976. 
[BPV] W. Barth, C. Peters and A. Van de Ven, Compact Complex Surfaces, Springer, Berlin, Heidelberg, New York, 1984.

[BH] P.J. Braam and J. Hurtubise, Instantons on Hopf surfaces and monopoles on solid tori, J. Reine Angew. Math., 400 (1989), 146-172.

[Br] R. Brussee, Stable bundles on blown up surfaces, Math. Z., 205 (1990), 551-565.

[B1] N.P. Buchdahl, Instantons on $\mathbb{C} P_{2}$, J. Differ. Geom., 24 (1986), 19-52.

[B2] _ Stable 2-bundles on Hirzebruch surfaces, Math. Z., 194 (1987), 143-152.

[B3] , Hermitian-Einstein connections and stable vector bundles over compact complex surfaces, Math. Ann., 280 (1988), 625-648.

[B4] _ Instantons on $n \mathbb{C P}_{2}$, J. Differ. Geom., 37 (1993), 669-687.

[B5] - Sequences of stable vector bundles over compact complex surfaces, J. Geom. An., 9 (1999), 391-428.

[D1] S.K. Donaldson, A new proof of a theorem of Narasimhan and Seshadri, J. Differ. Geom., 18 (1983), 269-277.

[D2] _ Anti-self-dual Yang-Mills connections over complex algebraic varieties and stable vector bundles, Proc. Lond. Math. Soc., 50 (1985), 1-26.

[D3] , Connections, cohomology and the intersection forms of 4-manifolds, J. Differ. Geom., 24 (1986), 275-341.

[D4] , Irrationality and the h-cobordism conjecture, J. Differ. Geom., 26 (1987), 141-168.

[D5] , Polynomial invariants for smooth four manifolds, Topology, 29 (1990), 257-315.

[FS] R. Fintushel and R.J. Stern, The blowup formula for Donaldson invariants, Ann. of Math., 143 (1996), 529-546.

[FM1] R. Friedman and J.W. Morgan, On the diffeomorphism types of certain algebraic surfaces I, J. Differ. Geom., 27 (1988), 297-369.

[FM2] - On the diffeomorphism types of certain algebraic surfaces II, J. Differ. Geom., 27 (1988), 371-398.

[FM3] _ Smooth Four-Manifolds and Complex Surfaces, Erg. Math. u. Grenzgebiete, 37(3), Springer, Berlin Heidelberg New York, 1994.

[Gau] P. Gauduchon, Le théorème de l'excentricité nulle, C. R. Acad. Sci. Paris, 285 (1977), 387-390.

[Gie] D. Gieseker, On the moduli of vector bundles on an algebraic surface, Ann. Math., 106 (1977), 45-60.

[K1] D. Kotschick, On manifolds homeomorphic to $\mathbb{C P}^{2} \# 8 \overline{\mathbb{C P}}^{2}$, Invent. Math., 95 (1989), 591-600.

[K2] $\quad S O(3)$ invariants for 4-manifolds with $b_{2}^{+}=1$, Proc. Lond. Math. Soc., 63 (1991), 426-448.

[L] M. Lübke, Chernklassen von Hermite-Einstein Vektor-Bündeln, Math. Ann., 260 (1982), 133-141.

[M] M. Maruyama, On a compactification of a moduli space of stable bundles on a rational surface, in 'Algebraic Geometry and Commutative Algebra', Kinokuniya, Tokyo, (1988), 233-260. 
[OSS] C. Okonek, M. Schneider and H. Spindler, Vector bundles on complex projective spaces, Birkhäuser, Boston, Basel, Stuttgart, 1980.

[OV] C. Okonek and A. Van de Ven, Stable bundles and differentiable structures on certain algebraic surfaces, Invent. Math., 86 (1986), 357-370.

[Sch] R.L.E. Schwarzenberger, Vector bundles on algebraic surfaces, Proc. Lond. Math. Soc., 11(3) (1961), 601-622.

[Sed] S. Sedlacek, A direct method for minimizing the Yang-Mills functional, Commun. Math. Phys., 86 (1982), 515-528.

[Ser] J.-P. Serre, Sur les modules projectifs, Sém. Dubreil-Pisot, Exposé 2 (1960/61).

[T] C.H. Taubes, Self-dual Yang-Mills connections on non-self-dual 4-manifolds, J. Differ. Geom., 17 (1982), 139-170.

[U1] K.K. Uhlenbeck, Connections with $L^{p}$ bounds on curvature, Commun. Math. Phys., 83 (1982), 31-42.

[U2] _ Removable singularities in Yang-Mills fields, Commun. Math. Phys., 83 (1982), 11-30.

Received June 30, 1997 and revised December 9, 1999. This research was partially supported by NSF Grant \#8900878.

UNIVERSity OF AdELAide

Adelaide, Australia 5005

E-mail address: nicholas.buchdahl@adelaide.edu.au 